\title{
The luminosity function of cluster galaxies
}

\section{Data reduction procedures applied to the cluster Abell $496^{\star}$}

\author{
A. Moretti ${ }^{1}$, E. Molinari ${ }^{1}$, G. Chincarini ${ }^{1,2}$, and S. De Grandi ${ }^{1}$ \\ 1 Osservatorio Astronomico di Brera, Via Bianchi 46, I-22055 Merate, Italy \\ 2 Università degli Studi di Milano, via Celoria 16, I-20133 Milano, Italy
}

Received March 23; accepted August 30, 1999

\begin{abstract}
We initiated a large project aimed to estimate the Luminosity Function of galaxies in clusters and to evaluate its relation to cluster morphology. With this paper we deem necessary to outline the general procedures of the data reduction and details of the data analysis. The cluster sample includes the brightest southern ROSAT all-sky survey clusters with $z<0.1$. These have been observed in three colours $g, r, i$, and mapped up to a few core radii using a mosaic of $\mathrm{CCD}$ frames. $\mathrm{E} / \mathrm{S} 0$ galaxies in the cluster core are singled out both by morphology (for the brightest galaxies), and by colour. The details of the data reduction procedure are illustrated via the analysis of the cluster Abell 496, which has been used as a pilot cluster for the whole program. The related photometric catalogue consists of 2355 objects. The limiting magnitudes (the reference Surface Brightness is given in parenthesis) in the various colours are respectively $g(25.5)=24.14$, $r(25.5)=24.46, i(25.0)=23.75$. These correspond to the limiting absolute magnitudes $-12.28,-11.96$ and -12.67 $\left(H_{0}=50 \mathrm{~km} / \mathrm{s} / \mathrm{Mpc}\right)$.
\end{abstract}

Key words: galaxies: photometry - galaxies: cluster: general

\section{Introduction}

In 1993/1994 we started a long-range photometry program on clusters of galaxies in order to estimate in detail the cluster Luminosity Function (LF) and the morphology of the brightest cluster galaxies. Our aim was to gain more accurate knowledge on this topic both to better understand formation and evolution, and to improve the comparison with numerical simulations. Straightforward

Send offprint requests to: moretti@merate.mi.astro.it

* Based on observation carried out at ESO La Silla, Chile. scientific drivers are at the basis of this investigation: the Luminosity Function of cluster galaxies at present time is the result of cluster initial formation and subsequent evolution - taking into account internal phenomena and external interactions.

It is reasonable, and to some extent expected, that at formation the galaxy mass function is a universal constant. In this case, assuming that every evolutionary process keeps a constant $\mathrm{M} / \mathrm{L}$ ratio, it would be reasonable to expect a universal LF, even if it cannot be excluded that evolution and richness might play a role on this stage. Present day observing evidence is, however, that the mass is organised into differently shaped and differently luminous galaxies - the galaxy population depending strongly on the cluster density and morphology. It would be strange if Nature, in the unfolding of this multivariate process, could set to work such a fine-tuning as to maintain the exact proportionality between mass and luminosity, even assuming a universal initial mass function.

The assumption of a universal LF for all the clusters (Colless 1989 and, more recently, Threntham 1997, 1998) might therefore be too coarse of a tool for characterizing the cluster population. Infall and ICM-galaxies interaction might further perturb the shape of the LF during the evolution of the cluster. In this respect it seemed of fundamental importance to evaluate the faint end of the LF. Meanwhile, important work has been published on this topic following the excellent papers on the Virgo Cluster by Binggeli et al. (1988). Biviano et al. (1995) approached the study by selecting a catalogue of bright galaxies in the Coma cluster. Undoubtedly, this direct method is a sound way to proceed, but the construction of a spectrophotometric catalogue of a large number of rich clusters demands an unaffordable amount of time with a 4 meter class telescope.

Another very interesting photometric approach is that of Bernstein et al. (1995), for the same Coma cluster, where particular attention has been given to the faint end 
of LF. In that work, however, the bright part remains illdefined.

A general consideration of the different studies is the limited application of their results, often making it impossible to compare directly the catalogue and Lfs. This led us to build our consistent photometric catalogues.

In this paper we outline at first the selected sample: other authors might be interested in this bookkeeping (avoiding or comparing duplications) and it will help the reader to follow our work to its the completion.

Secondly, we detail our observational strategy and methods of data reduction, particularly in those points where they differ from the standard analysis used in the literature. They will then form a basic reference for other papers in preparation. The observing strategies are strongly related and tuned to the data analysis methods. These procedures have first been applied to the cluster Abell 496 (see also Molinari et al. 1998, Paper I, for discussion on LF), for which we publish here the photometry.

\section{The project}

\subsection{The sample}

The sample has been selected from the catalogue given in De Grandi et al. (1999) by choosing only clusters at declination $<0^{\circ}$, with X-ray fluxes measured in the $0.5-$ $2.0 \mathrm{keV}$ energy band larger than $10^{-11} \mathrm{erg} \mathrm{cm}^{-2} \mathrm{~s}^{-1}$, and with extended X-ray emission (i.e., sources with probability to be point-like, as computed by De Grandi et al. 1997, smaller than 1\%). The resulting sample of 20 clusters is reported in Table 1. Columns list the main X-ray and optical properties for each source as follows: Column (1) - Cluster name. Column (2) - X-ray position: J2000.0 right ascension (hh mm ss.s). Column (3) - X-ray position: J2000.0 declination (dd mm ss.s). Column (4) Cluster red-shift. Column (5) — Unabsorbed X-ray flux computed in the $0.5-2.0 \mathrm{keV}$ band in units of $10^{-11} \mathrm{erg}$ $\mathrm{cm}^{-2} \mathrm{~s}^{-1}$. Column (6) - Bautz-Morgan type. Column (7) - Optical richness. Column (8) - Status of observations (Obs. = observed).

\subsection{Imaging}

CCD observations of the sample clusters were carried out since December 1994 at La Silla with the $1.5 \mathrm{~m}$ Danish Telescope equipped with DFOSC camera. For each cluster we observed a mosaic composed of 3 or 4 slightly overlapping fields (Fig. 2 shows the mosaic of Abell 496). In each mosaic the centre of the first field corresponds to the centre of the X-ray isophotes (see the Fig. 1 in Molinari et al. 1998). The other fields are centred along a radial direction. For each mosaic, the typical total observed area is $250 \operatorname{arcmin}^{2}$ with a typical maximum angular distance of $30 \mathrm{arcmin}$ (equal to a linear distance of $2.5 \mathrm{Mpc}$ at

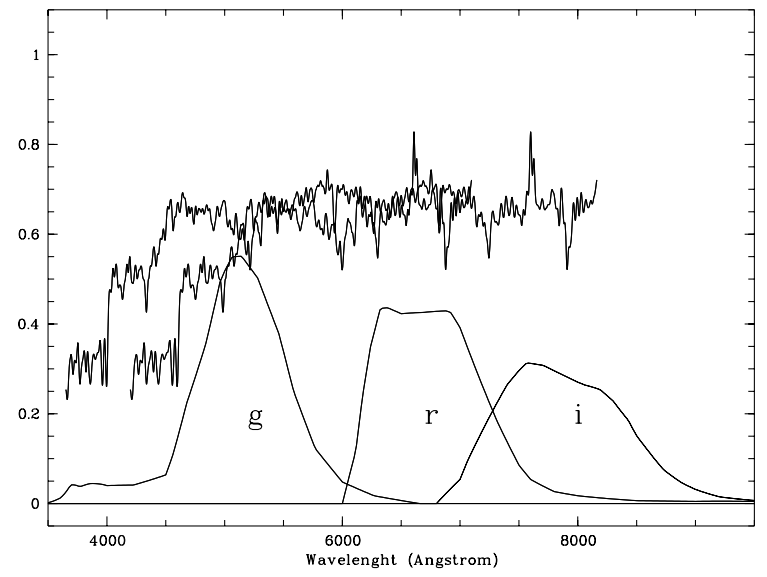

Fig. 1. The efficiency of the $g, r, i$ filters as function of the wavelength. In comparison, a typical early type galaxy spectrum is superimposed at two different red-shifts: $z=0$ and $z=0.12$, the extremes of the catalogue redshift range

$z=0.05)$. For each cluster the observation of the most external field is used mainly to evaluate the background. Each field is observed with the $g, r, i$ filters of the Gunn photometric system (Thuan \& Gunn 1976; Wade et al. 1979). The spectral response is illustrated in Fig. 1 along with the observed spectrum of an elliptical galaxy.

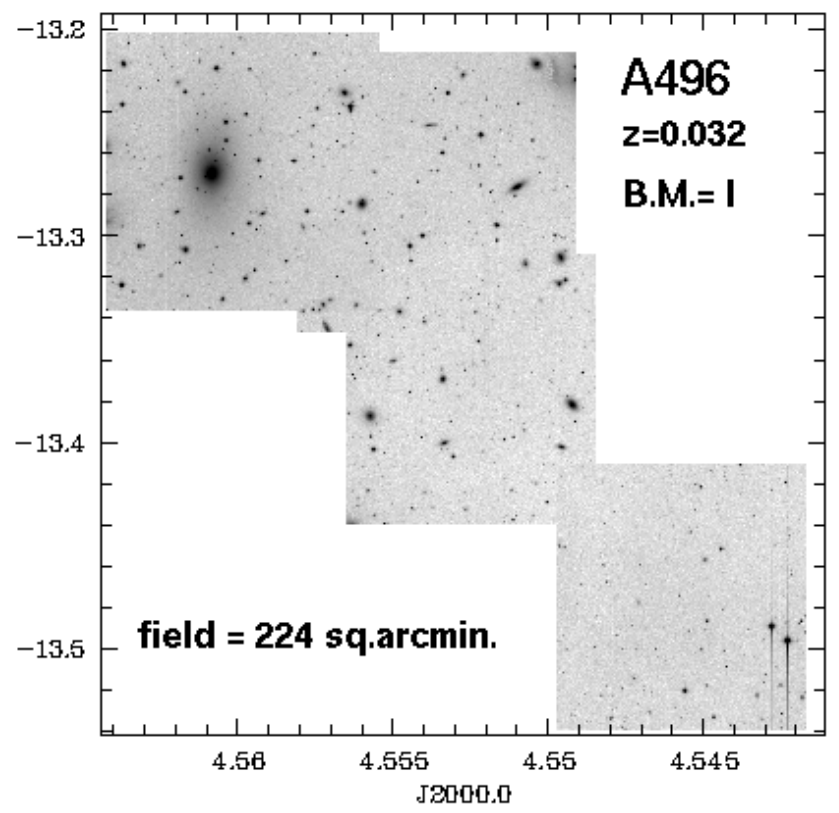

Fig. 2. The observed field of the cluster Abell 496 with the $c D$ galaxy in the NE corner of the image. The mosaic is composed of 4 adjoining, and slightly overlapping fields: their identification number (Table 2) increases moving from NE (field 0) to $\mathrm{SO}$ corner (field 3). The angular distance between $\mathrm{NE}$ and $\mathrm{SO}$ corners is 30 arcmin 
Table 1. The sample. Data relative to X-ray flux and red-shift are from De Grandi et al. (1999). Data relative to optical richness and morphology are from Abell et al. (1989). Data labelled with * are our estimate

\begin{tabular}{|c|c|c|c|c|c|c|c|}
\hline Name & $\alpha_{2000}$ & $\delta_{2000}$ & Redshift & Flux & B.M. & Rich. & Status \\
\hline A0085 & $00 \mathrm{~h} 41 \mathrm{~m} 50.11 \mathrm{~s}$ & $-09 \mathrm{~d} 18 \mathrm{~m} 17.5 \mathrm{~s}$ & 0.05560 & $4.092_{-0.244}^{+0.256}$ & I & 2 & Obs. \\
\hline A0119 & $00 \mathrm{~h} 56 \mathrm{~m} 11.69 \mathrm{~s}$ & $-01 \mathrm{~d} 14 \mathrm{~m} 52.5 \mathrm{~s}$ & 0.04420 & $2.406_{-0.708}^{+0.854}$ & II-III & 2 & Obs. \\
\hline A0133 & $01 \mathrm{~h} 02 \mathrm{~m} 42.21 \mathrm{~s}$ & $-21 \mathrm{~d} 52 \mathrm{~m} 43.5 \mathrm{~s}$ & 0.05660 & $1.578_{-0.166}^{+0.181}$ & $\mathrm{I}^{*}$ & 2 & Obs. \\
\hline A3158 & $03 \mathrm{~h} 42 \mathrm{~m} 53.06 \mathrm{~s}$ & $-53 \mathrm{~d} 37 \mathrm{~m} 43.0 \mathrm{~s}$ & 0.05910 & $2.250_{-0.184}^{+0.206}$ & I-II & 2 & - \\
\hline A3186 & $03 \mathrm{~h} 52 \mathrm{~m} 25.09 \mathrm{~s}$ & $-74 \mathrm{~d} 01 \mathrm{~m} 02.5 \mathrm{~s}$ & 0.12700 & $1.041_{-0.157}^{+0.177}$ & I-II & 1 & Obs. \\
\hline EXO0422-086 & $04 \mathrm{~h} 25 \mathrm{~m} 51.02 \mathrm{~s}$ & $-08 \mathrm{~d} 33 \mathrm{~m} 38.5 \mathrm{~s}$ & 0.03971 & $\begin{array}{l}1.870_{-0.179}^{+0.188} \\
\end{array}$ & $I^{*}$ & - & Obs. \\
\hline A 3266 & 04h30m58.82s & $-61 \mathrm{~d} 27 \mathrm{~m} 52.5 \mathrm{~s}$ & 0.05890 & $3.001_{-0.717}^{+0.869}$ & I & 2 & Obs. \\
\hline A0496 & $04 \mathrm{~h} 33 \mathrm{~m} 37.07 \mathrm{~s}$ & $-13 \mathrm{~d} 15 \mathrm{~m} 20.0 \mathrm{~s}$ & 0.03284 & $4.652_{-0.389}^{+0.423}$ & I & 1 & Obs. \\
\hline A 3376 & $06 \mathrm{~h} 01 \mathrm{~m} 37.77 \mathrm{~s}$ & $-40 \mathrm{~d} 00 \mathrm{~m} 31.0 \mathrm{~s}$ & 0.04550 & $1.504_{-0.429}^{+0.519}$ & I & 1 & - \\
\hline A3391 & $06 \mathrm{~h} 26 \mathrm{~m} 20.10 \mathrm{~s}$ & $-53 \mathrm{~d} 41 \mathrm{~m} 44.5 \mathrm{~s}$ & 0.05310 & $1.313_{-0.152}^{+0.183}$ & I & 0 & - \\
\hline A3395 & $06 \mathrm{~h} 27 \mathrm{~m} 38.83 \mathrm{~s}$ & $-54 \mathrm{~d} 26 \mathrm{~m} 38.5 \mathrm{~s}$ & 0.04980 & $1.122_{-0.209}^{+0.289}$ & I & 3 & Obs. \\
\hline A 3667 & $20 \mathrm{~h} 12 \mathrm{~m} 35.08 \mathrm{~s}$ & $-56 \mathrm{~d} 50 \mathrm{~m} 30.5 \mathrm{~s}$ & 0.05560 & $3.289_{-0.668}^{+0.927}$ & II & 2 & Obs. \\
\hline A3695 & $20 \mathrm{~h} 34 \mathrm{~m} 46.86 \mathrm{~s}$ & $-35 \mathrm{~d} 49 \mathrm{~m} 07.5 \mathrm{~s}$ & 0.08930 & $1.247_{-0.243}^{+0.315}$ & I & 2 & Obs. \\
\hline A 3822 & $21 \mathrm{~h} 54 \mathrm{~m} 10.21 \mathrm{~s}$ & $-57 \mathrm{~d} 52 \mathrm{~m} 05.5 \mathrm{~s}$ & 0.07590 & $1.099_{-0.170}^{+0.202}$ & II-III & 2 & Obs. \\
\hline A 3827 & $22 \mathrm{~h} 01 \mathrm{~m} 58.85 \mathrm{~s}$ & $-59 \mathrm{~d} 57 \mathrm{~m} 37.0 \mathrm{~s}$ & 0.09840 & $1.517_{-0.160}^{+0.170}$ & I & 2 & - \\
\hline A 2420 & $22 \mathrm{~h} 10 \mathrm{~m} 20.09 \mathrm{~s}$ & $-12 \mathrm{~d} 10 \mathrm{~m} 49.0 \mathrm{~s}$ & 0.08380 & $1.172_{-0.199}^{+0.240}$ & I & 2 & Obs. \\
\hline A3921 & $22 \mathrm{~h} 50 \mathrm{~m} 03.61 \mathrm{~s}$ & $-64 \mathrm{~d} 26 \mathrm{~m} 30.0 \mathrm{~s}$ & 0.09360 & $1.201_{-0.226}^{+0.259}$ & II & 2 & Obs. \\
\hline RX-J2344.2-422 & $23 \mathrm{~h} 44 \mathrm{~m} 15.98 \mathrm{~s}$ & $-04 \mathrm{~d} 22 \mathrm{~m} 24.5 \mathrm{~s}$ & 0.07860 & $1.214_{-0.232}^{+0.302}$ & $\mathrm{I}^{*}$ & - & - \\
\hline A4038 & $23 \mathrm{~h} 47 \mathrm{~m} 41.78 \mathrm{~s}$ & $-28 \mathrm{~d} 08 \mathrm{~m} 26.5 \mathrm{~s}$ & 0.02920 & $2.751_{-0.221}^{+0.232}$ & III & 2 & Obs. \\
\hline A4059 & $23 \mathrm{~h} 57 \mathrm{~m} 00.02 \mathrm{~s}$ & $-34 \mathrm{~d} 45 \mathrm{~m} 24.5 \mathrm{~s}$ & 0.04600 & $1.974_{-0.178}^{+0.189}$ & I & 1 & Obs. \\
\hline
\end{tabular}

Observations of each field consist of $3600 \mathrm{~s}$ exposure as a result of the integration of $4 \times 900 \mathrm{~s}$ different exposures. Up to date, we have collected photometric observations of 15 out of 20 clusters of the sample (Table 1 ). Spectroscopic observations are also being planned and will likely start shortly before completion of the photometric sample. This paper will deal, in particular, with the data analysis carried out for the cluster Abell 496. However it reflects the method we will also use for the other clusters.

\section{Abell 496 image processing}

Abell 496 is a class 1 rich cluster, Bautz Morgan type I (Abell et al. 1989), dominated by a single central cD galaxy, MGC -02-12-039 $\left(\alpha_{2000}=4^{h} 33^{\prime} 37.7^{\prime \prime}\right.$, $\left.\delta_{2000}=-13^{\circ} 15^{\prime} 43.2^{\prime \prime}, z=0.032\right)$. The peak of the X-ray emission lies inside the core of the cD galaxy (Table 1). CCD observations of the cluster were carried out during the first observing run from 24 to 27 December 1994. The effective field of the DFOSC camera and Thomson THX 31156 CCD is $8.68 \times 8.68$ arcmin with a single pixel corresponding to 0.508 arcsec. The total area of the observed field is $224 \operatorname{arcmin}^{2}$ for each filter (Fig. 2). We list the journal of the observations in Table 2 .

\subsection{Flat-fielding and magnitude calibration}

Basic data reduction, including bias subtraction, flatfield correction, magnitude calibration and cosmic rays subtraction, is done using the ESO-MIDAS software environment.

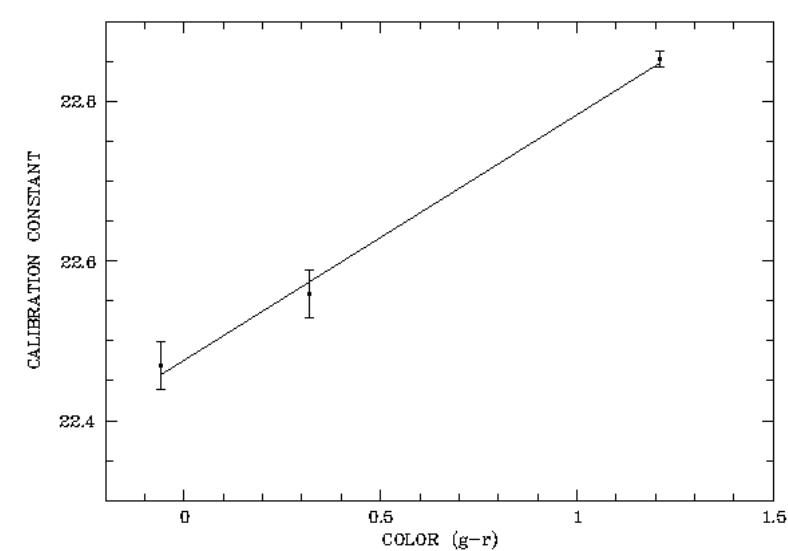

Fig. 3. The calibration straight line for filter $r$. For each of the three standard star, the typical uncertainty on the offset measure is 0.02 magnitude. Moreover, the 3 different average offset values show a linear dependence on the colour of the star. By the linear fit, we extrapolate the offset value corresponding to $g-r=0$

For each filter we build two different flat-field frames. For the first we use the dithering method to obtain the flat field frame directly from scientific exposures (see for example Molinari et al. 1996). The second flat field frame is built using the median of the distribution of the sunset and twilight sky images. We obtain the minimum value of the ratio noise/sky, at both small and big scales in the frames, using the first flat-fielding procedure for filter $i$. For the $g$ and $r$ filters we adopt the average between the two different flat-field frames, since this gives the minimum rms. After the reduction, the typical rms of the 
Table 2. The journal of observations. The Date, Universal Time, air mass, exposure time, and seeing for each frame are shown. In each frame seeing is calculated as the FWHM of the stars

\begin{tabular}{|c|c|c|c|c|c|c|}
\hline Object & Filter & Date & U.T. & airmass & E.T. & $\begin{array}{l}\text { Seeing } \\
\end{array}$ \\
\hline \multirow[t]{12}{*}{ field0 } & \multirow[t]{4}{*}{$g$} & $25-12-94$ & $1: 54$ & 1.071 & \multirow{4}{*}{$\begin{array}{l}900 \mathrm{~s} . \\
", \\
"\end{array}$} & 1.25 \\
\hline & & & $2: 48$ & 1.040 & & 1.25 \\
\hline & & & $3: 41$ & 1.060 & & 1.50 \\
\hline & & & $4: 33$ & 1.133 & & 1.50 \\
\hline & \multirow[t]{4}{*}{$r$} & & $1: 37$ & 1.092 & \multirow[b]{2}{*}{$"$} & 1.25 \\
\hline & & & 3:05 & 1.041 & & 1.25 \\
\hline & & & $3: 24$ & 1.048 & $"$ & 1.50 \\
\hline & & & $4: 50$ & 1.171 & & 1.50 \\
\hline & \multirow{4}{*}{\multicolumn{2}{|c|}{$i$}} & $2: 12$ & 1.054 & & 1.25 \\
\hline & & & $2: 31$ & 1.044 & $"$ & 1.25 \\
\hline & & & $3: 58$ & 1.077 & $"$ & 1.50 \\
\hline & & & $4: 16$ & 1.102 & $"$ & 1.50 \\
\hline \multirow{12}{*}{ field1 } & \multirow[t]{4}{*}{$g$} & \multirow{4}{*}{$26-12-94$} & $1: 45$ & 1.076 & \multirow{4}{*}{$\begin{array}{l}" 1 \\
" \\
"\end{array}$} & 1.50 \\
\hline & & & $2: 14$ & 1.050 & & 1.50 \\
\hline & & & $3: 41$ & 1.063 & & 1.15 \\
\hline & & & 4:06 & 1.093 & & 1.15 \\
\hline & \multirow[t]{4}{*}{$r$} & & $1: 12$ & 1.128 & \multirow{4}{*}{$\begin{array}{l}" 1 \\
" \\
"\end{array}$} & 1.50 \\
\hline & & & $2: 32$ & 1.042 & & 1.50 \\
\hline & & & $3: 24$ & 1.050 & & 1.15 \\
\hline & & & $4: 23$ & 1.121 & & 1.15 \\
\hline & \multirow{4}{*}{\multicolumn{2}{|c|}{$i$}} & $1: 28$ & 1.100 & \multirow{4}{*}{$\begin{array}{l}" \\
" \\
"\end{array}$} & 1.50 \\
\hline & & & $2: 50$ & 1.039 & & 1.50 \\
\hline & & & $3: 07$ & 1.042 & & 1.15 \\
\hline & & & $4: 40$ & 1.157 & & 1.15 \\
\hline \multirow[t]{12}{*}{ 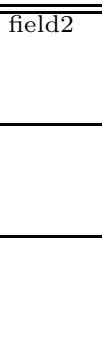 } & \multirow[t]{4}{*}{$g$} & $27-12-94$ & $\overline{1: 21}$ & $\overline{1.105}$ & \multirow{4}{*}{$\begin{array}{l}" \\
" \\
"\end{array}$} & 1.30 \\
\hline & & & $5: 04$ & 1.233 & & 1.35 \\
\hline & & & $5: 22$ & 1.298 & & 1.35 \\
\hline & & $28-12-94$ & $1: 49$ & 1.064 & & 1.25 \\
\hline & \multirow[t]{4}{*}{$r$} & $27-12-94$ & $1: 05$ & 1.134 & \multirow{4}{*}{$\begin{array}{l}" \\
" \\
"\end{array}$} & 1.30 \\
\hline & & & $4: 47$ & 1.184 & & 1.35 \\
\hline & & & $5: 39$ & 1.372 & & 1.35 \\
\hline & & $28-12-94$ & $1: 32$ & 1.083 & & 1.25 \\
\hline & \multirow[t]{4}{*}{$\bar{i}$} & $27-12-94$ & $1: 38$ & 1.080 & & 1.30 \\
\hline & & & $1: 56$ & 1.061 & $"$ & 1.30 \\
\hline & & $28-12-94$ & $0: 58$ & 1.140 & $"$ & 1.25 \\
\hline & & & $1: 16$ & 1.106 & $"$ & 1.25 \\
\hline field3 & $g$ & $28-12-94$ & $2: 14$ & 1.046 & $900 \mathrm{~s}$. & 1.25 \\
\hline & & & $4: 11$ & 1.114 & & 1.50 \\
\hline & & & $5: 35$ & 1.371 & $"$ & 1.50 \\
\hline & & & $5: 52$ & 1.462 & $600 \mathrm{~s}$. & 1.50 \\
\hline & $r$ & & $2: 31$ & 1.040 & $900 \mathrm{~s}$. & 1.25 \\
\hline & & & $4: 28$ & 1.147 & $"$ & 1.50 \\
\hline & & & $5: 18$ & 1.297 & $"$ & 1.50 \\
\hline & & & $6: 04$ & 1.538 & $600 \mathrm{~s}$. & 1.50 \\
\hline & $i$ & & $2: 48$ & 1.040 & 900s. & 1.25 \\
\hline & & & $4: 45$ & 1.189 & $"$ & 1.50 \\
\hline & & & $5: 02$ & 1.240 & $"$ & 1.50 \\
\hline & & & $6: 15$ & 1.618 & $600 \mathrm{~s}$. & 1.50 \\
\hline
\end{tabular}

sky is $1.5 \%, 1 \%, 0.75 \%$ of the background for the $g, r$, $i$ frames, respectively. Cosmic rays are identified by their appearance in only one of the dithered images. The stars observed as standard are selected in the photometric system of Thuan \& Gunn (1976) and are listed in Table 3. The offset of the calibration is measured as the difference between instrumental magnitude (as measured with the $g, r, i$ filters at ESO telescope) and the magnitude of the standard stars. In spite of the fact that we evaluate a relation between the colour of the standard star and the magnitude off-set, we decided not to account for the colour relation due to the paucity of the data and the possibility of systematic errors. Most importantly, we could not apply the colour correction to galaxies which have been detected only in one or two filters ( $40 \%$ of the sample). Choosing the best compromise, we applied in all cases the
Table 3. Standard stars used for calibration

\begin{tabular}{|c|c|c|c|c|c|}
\hline Name & $\alpha_{1950}$ & $\delta_{1950}$ & $g$ & $r$ & $i$ \\
\hline HD 84937 & $\begin{array}{llll}09 & 46 & 12.1\end{array}$ & +135917.0 & 8.325 & 8.383 & 8.43 \\
\hline Ross 683 & 084746.6 & +074908.0 & 11.40 & 11.08 & - \\
\hline $\mathrm{BD}-15^{\circ} 6290$ & $2250 \quad 37.5$ & -143142.0 & 10.754 & 9.544 & 8.334 \\
\hline
\end{tabular}

magnitude correction equivalent to $g-r=0$. Because of this assumption, our photometric data differ slightly from the photometric Gunn system (typically 0.1 magnitude for an object with $g-r=1$ ). The colours we measure, however, match very well the Gunn system, because the slopes of the calibration straight lines in the three filters are similar.

Table 4. k correction (Buzzoni 1995) and galactic extinction (Burstein \& Heiles 1982) values used for the E/S0 galaxies in Abell 496

\begin{tabular}{|l|ccc|}
\hline filter & $g$ & $r$ & $i$ \\
\hline k corr. & 0.02 & 0.01 & 0.01 \\
gal. ext. & 0.07 & 0.04 & 0.03 \\
\hline
\end{tabular}

\subsection{Object search and analysis}

Automatic object detection and magnitude evaluation have been done by using the INVENTORY package (West \& Kruszewski 1981) implemented in the MIDAS environment. Galaxies of the sample span a very large range in magnitude from the magnitude limit (mag $\sim 24$, see next section) to the isophotal magnitude (mag $\sim 13$ ) of the $\mathrm{cD}$ central galaxy. This range corresponds to a comparable range in the size of the galaxies. It varies from the PSF limit ( $\sim 3$ pixels $)$ to the isophotal radius of the $\mathrm{cD}$ galaxy $(\sim 100$ pixels $)$. Because of this inherent heterogeneity, the sample is not perfectly suitable for automatic search and analysis of the sources. In particular, we must separate the signal of very extended objects from the rest of the image to avoid the problem of multiple detection. The procedure we use is composed of the following three points. First, we model and subtract the light of the most extended objects. Second, we apply the INVENTORY standard research and analysis procedure to frames in which the remaining objects are comparable in size. Finally, we apply the INVENTORY analysis routine to the single-object images of the modelled and rebuilt extended objects. Here we describe only the first point of the procedure which is the original part. We model and rebuild the extended sources, typically giant elliptical galaxies, with a procedure similar to the one described by Molinari et al. (1996). We improved their algorithm by making it more flexible. First, for each distance from the centre of the galaxy, the algorithm analyses the azimuthal intensity profile along the circular paths (see the left panel in Fig. 4). The projection 

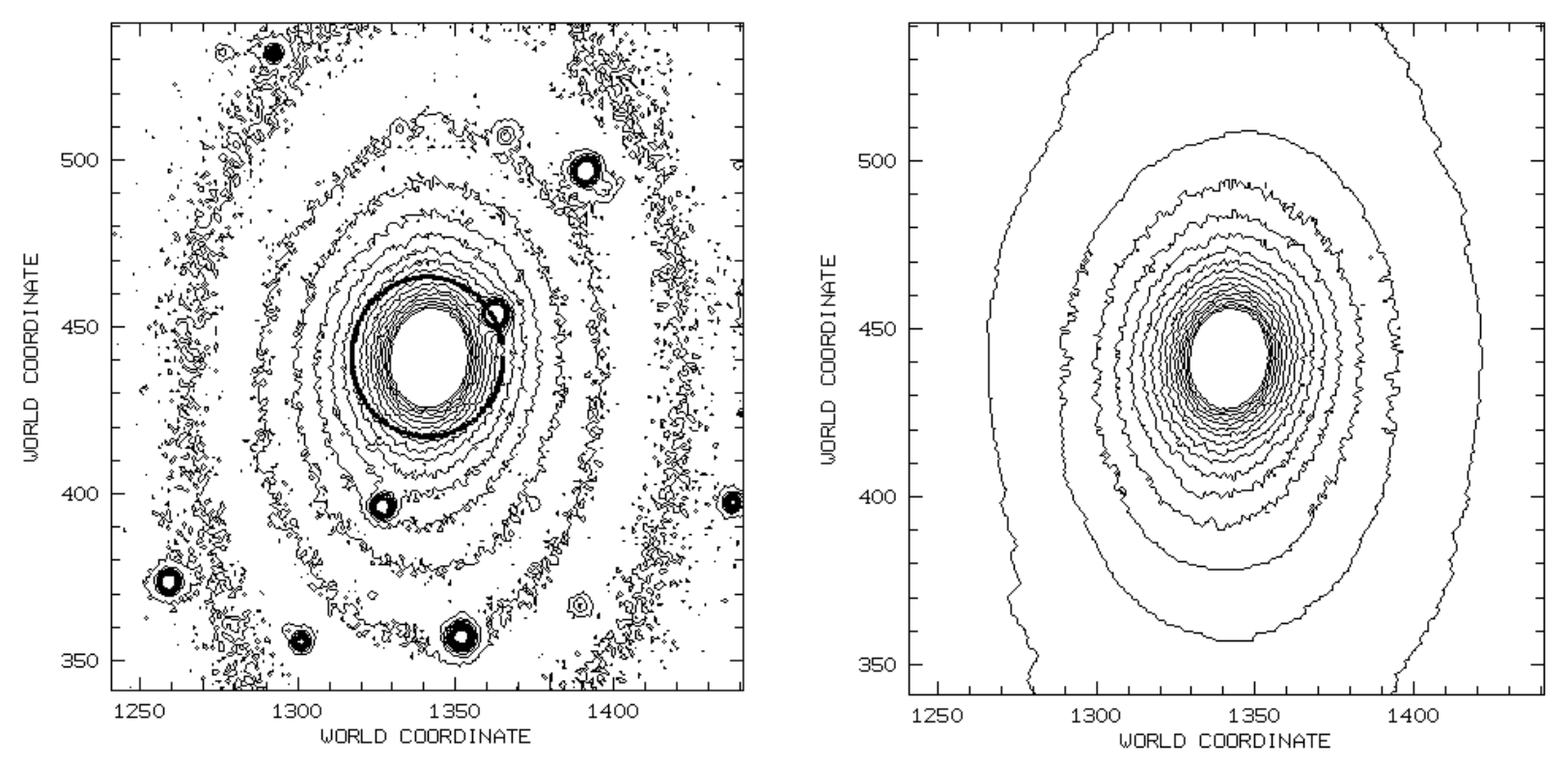

Fig. 4. Isophotes of the cD galaxy of Abell 496 from the raw image (left panel), and from the rebuilt model (right panel). The coordinate refer to the pixels of the image: 1 pixel $=0.508$ arcsec. In the left panel the circular path at 24 pixel radius is marked; the intensity profile along this path is reported in the panel A of Fig. 5. The model is built using raw data where possible and fit value when an external object is superimposed on the line of sight

of an elliptical isophote on the circular paths yields a periodic variation of surface brightness, as shown in the panel A of Fig. 5. It corresponds to the intensity profile along the circular path marked on the left panel of Fig. 4. The maxima correspond to the intersections of the circular path with the major semi-axis of the isophote. Then the algorithm fits the profile using a Fourier series and a low-pass filter. This procedure eliminates the physical and geometrical high frequency noise due to the discrete nature of the CCD pixel grid. Finally, we calculate the distribution of the differences between the data and the fit: we exclude from the profile the points whose intensity is greater than 3 times the standard deviation of the distribution (Fig. 5, panel B). Those points are replaced by the exact fit values. By iterating a few times the procedure, we can separate the signals of the superimposed sources (Fig. 5 panels C), without any assumption on the shape of the isophotes. We also made the algorithm more flexible by introducing other geometrical parameters. In particular, we allow for the exclusion of selected angular profiles intervals from the calculation of the Fourier coefficient of the trigonometric series. Intervals to be excluded are selected by visual inspection. The exclusion option is useful when two objects of comparable size overlap and have very close intensity maxima. In this way, we can rebuild the hidden isophothes assuming a central symmetry. In Fig. 4 we compare the isophotes of the raw image of the $\mathrm{cD}$ galaxy (left panel) with the rebuilt model (right panel). The rebuilt model is then subtracted from the original frame to keep the photometric analysis of very extended sources separated.
Although time-consuming (due to its interactiveness), this procedure yields accurate photometric measurements of both the extended and small sources. The described procedure, in fact, allows the complete photometric analysis of the surface brightness of the extracted objects (see Sect. 5.1 for the Abell $496 \mathrm{cD}$ ). Contrary to other popular automated programs (e.g. SExtractor, Bertin \& Arnout 1996), we do not assign a pixel and its value to a unique object, but partition the flux in each pixel among the different objects detected. Thus the isophotes are recovered in their shape and intensity for all sources.

\section{The catalogue of Abell 496}

\subsection{Isophotal magnitude definition}

To define properly an isophotal magnitude we first need to consider some definitions and correlations (see also Trentham 1997).

\subsubsection{Isophotal versus total magnitude}

The difference between total and isophotal magnitude is the difference between the total flux, extrapolation of the curve of growth, and the flux integrated within a fixed SB value. To simulate such difference, we extract from the frames some bright sources ( $\sim$ magnitude 16 ) of different morphological types and integrate the total flux on an extrapolated model. We then increase the magnitude up to 


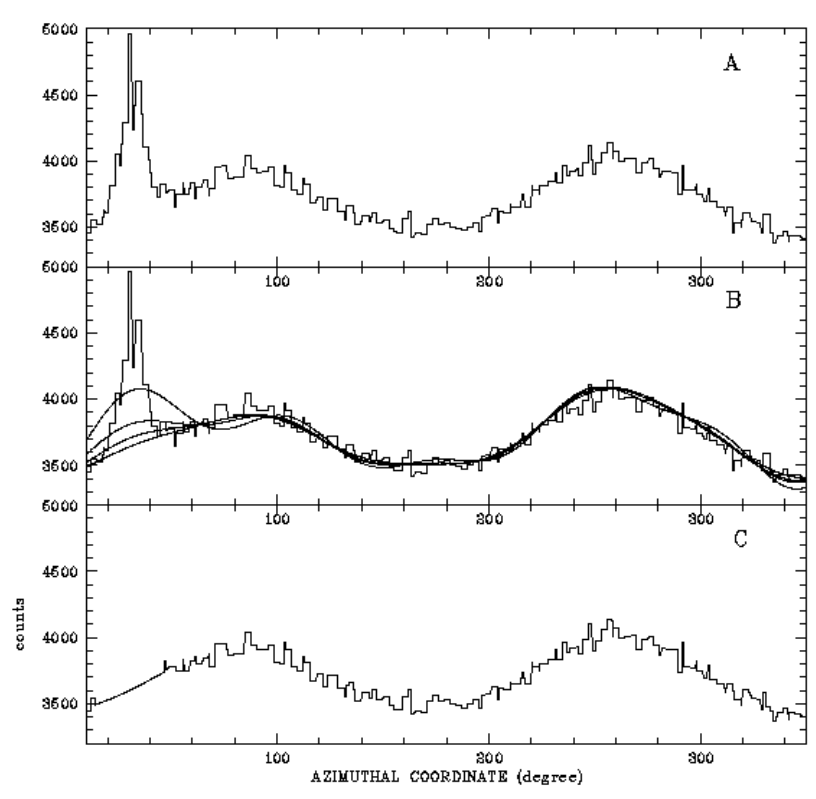

Fig. 5. Steps of the modelling procedure. A). The raw elliptical isophote is projected on a circular path. The profile shown here corresponds to the 24 pixel radius of the $\mathrm{cD}$ galaxy of Abell 496 as shown with a marked line in the left panel of Fig. 4. The azimuthal coordinate has the zero point toward the right of the image, and it increases counterclockwise. The periodic shape of period $\pi$ of the profile is evident: the two maxima are at 90 and 270 degrees, corresponding to the intersections between the path and the major axis of the elliptical isophotes (see Fig. 4). The profile of a superimposed source is evident at 30 degrees as a departure from the periodic shape. We can find the superimposed object along the path marked in Fig. 4 at 30 degrees from the 0 point of the azimuthal coordinate. The high frequency noise in the profile shape is due both to Poissonian and geometrical noises. B). Fit procedure is performed repeatedly excluding step by step the external object identified at $3 \sigma . \mathbf{C}$ ). When an external object is identified, the extended object is rebuilt using the fit value. Otherwise, the profile is left untouched

our frame limits by dividing the original flux by a numerical coefficient. In this way, we obtain a list of expected total magnitudes in the range of interest. We compare these values with the isophotal magnitudes as measured by the analysis routine with the threshold listed below. The amplitude of the differences is dependent on the source profile. In our data at $r \sim 24$ the differences range from $0.1 \mathrm{mag}$ for point like sources to few tenth of mag for E0/E6 galaxies and, little more than a magnitude for disk dominated objects (Fig. 6 shows the case of an elliptical$r^{1 / 4}$-galaxy). The difference is seeing dependent. To show this dependence we convolve the original frames (seeing $\simeq$ 1.3 arcsec) with a Gaussian point spread function to simulate worse seeing $(1.6$ arcsec). The effect is illustrated in Fig. 6.

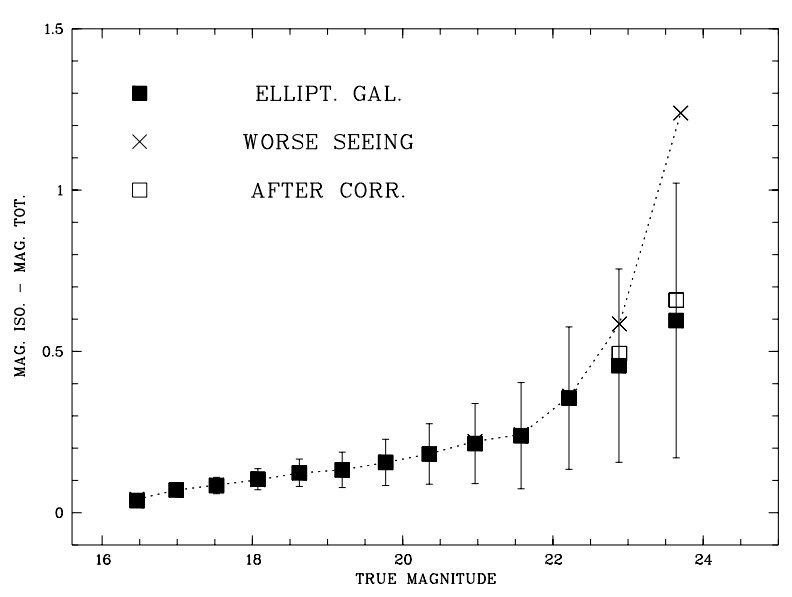

Fig. 6. The differences between the isophotal magnitude and the total magnitude of an elliptical galaxy (seeing = 1.3 arcsec) are plotted (filled squares) versus the total magnitude. Dashed line and crosses show the feature of the same elliptical galaxy with an artificially degraded seeing (1.6 arcsec). Open squares show the seeing-degraded galaxy after the correction performed according to the relationship seeing-threshold

\subsubsection{Dependence on the seeing}

To reach internal consistency on frames obtained with different seeing we must correct the isophotal magnitudes for the seeing of each frame. Our approach is as follows. We choose not to apply directly any correction to the isophotal magnitude, but, varying the value of the SB of the last isophote as a function of the seeing of the frame, we ensure that the isophotal magnitude value of a fixed morphological type always corresponds to the same fraction of the total flux of the source. The procedure is easily justified. Consider, for simplicity, a source with a Gaussian spatial brightness profile: in this case different seeing levels correspond to different values of the standard deviation $\sigma$ (Fig. 7) and the problem has a simple analytical solution. Let us consider a bidimensional symmetric Gaussian profile $I_{1}$ with $\sigma=\sigma_{1}$; given the threshold $\Sigma_{1}$ we have to consider the flux $\mathcal{F}$ subtended by $I_{1}$ from 0 to $r_{1}$, such that $I_{1}\left(r_{1}\right)=\Sigma_{1}$ :

$\mathcal{F}=\frac{1}{2 \pi \sigma^{2}} \int_{0}^{r_{1}} \mathrm{e}^{\frac{-r^{2}}{2 \sigma^{2}}} 2 \pi r \mathrm{~d} r$.

After the integration, we can write it as function of $\Sigma_{1}$

$\mathcal{F}=1-2 \pi \sigma_{1}^{2} \Sigma_{1}$.

Therefore given a different $\sigma=\sigma_{2}$ (and the same normalization), the same isophotal flux $\mathcal{F}$ is obtained using the threshold $\Sigma_{2}$ such that

$\Sigma_{2}=\left(\frac{\sigma_{1}}{\sigma_{2}}\right)^{2} \Sigma_{1}$.

We conventionally assume a limit surface brightness value as threshold in a frame with a certain seeing value and 
we use the relationship (1) to find the correct threshold in the other frames. The reference values of the limiting isophote SB are 25.5, 25.5, $25.0 \mathrm{mag} / \operatorname{arcsec}^{2}$ for $g, r, i$ filters, respectively with PSF $=1.3$ arcsec.

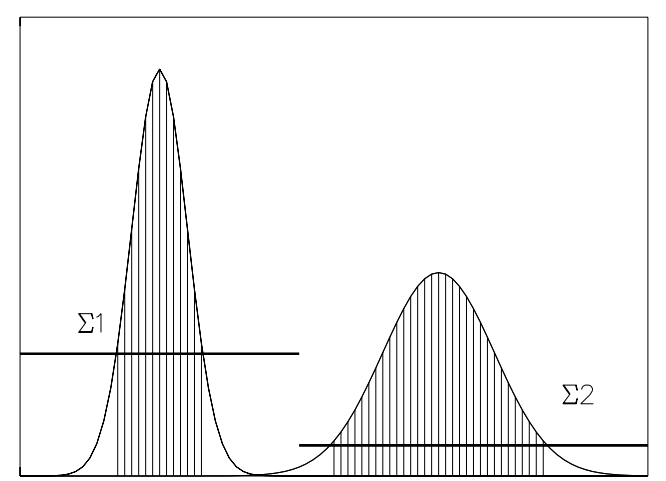

Fig. 7. The two Gaussian profiles simulate the same object observed with different PSF. The profiles are the projections of two bidimensional profiles with the same normalization and different FWHM. The marked areas represent the same quantity of flux. They represent the isophotal fluxes with different thresholds at two different seeing levels. According to Eq. (1), the second threshold $\Sigma_{2}$ is chosen in a such a way that the isophotal flux of the left profile is kept constant

The relation (1) has been deduced in the case of Gaussian profile source. We find that the corrections drawn from (1) give good results also for different morphological types as shown in Figs. 6 and 8. In Fig. 6 we show, in the case of an elliptical- $r^{1 / 4}$ galaxy, the difference between isophotal and true magnitude at two different seeing levels (one artificially degraded), and the difference after the correction. At low luminosity the correction substantially removes the seeing dependence.

The quality of the correction discussed above can be tested in the intersection regions of two overlapping frames, which have been obtained in different seeing conditions. In this region we have 2 different measures performed with different seeing of a list of sources of random magnitude and morphological type. For the differences between the 2 independent measures, we expect a symmetric distribution with a dispersion exponentially increasing with the magnitude due to the Poissonian uncertainty. If we remove this dependence by normalizing by an exponential factor, we expect a Gaussian distribution. In Fig. 8 we can observe that the distribution of the measures performed with the same threshold is slightly asymmetric; after adopting the threshold corrected according the relation (1) we find that the distribution of differences is perfectly symmetric as a test of reliability of the method described.

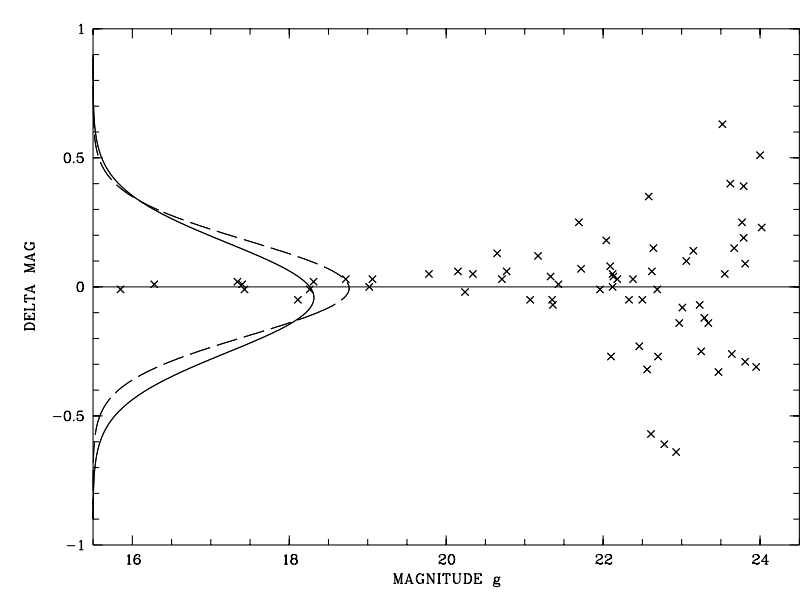

Fig. 8. The distribution of the differences between 2 measures with different seeing (1.3 arcsec vs. 1.4 arcsec) of 75 sources after the correction. The distribution of the differences of the measures before the correction is shown with the solid line and it is slightly asymmetric. The dashed line shows the symmetric distribution after the correction

\subsection{Sample completeness}

Background statistical variations and source crowding may affect the accuracy of the automatic detection routine and the completeness of the photometric catalogue. We use a bootstrapping technique to test the sensitivity of our results to both factors. First, we extract the image of a giant elliptical galaxy from one of the frames. Then, dividing by a numerical coefficient, we generate a set of more than 30 different images for each filter in the relevant range of isophotal magnitudes: $16.07 \leq r \leq 24.56,15.86 \leq g \leq$ $24.85,15.87 \leq i \leq 24.01$. The test images are added to the observed frames positioned at 25 subsequent distances from the centre of the cluster (assumed to be in the centre of $\mathrm{cD}$ galaxy). For each value of the distance from the centre and magnitude, we repeat this procedure 100 times in each filter, randomly changing the angular coordinate of the added test image. These 100 repetitions are divided in small groups in different runs to avoid bias due to artificial additional crowding. This allows us to estimate the probability of detecting a galaxy of magnitude $m$ at distance $r$ from the centre of the cluster $P(r, m)$. For each $P(r, m)$ we estimate the uncertainty by the binomial distribution $P_{B}[x, 100, P(r, m)]$, which gives the probability of observing $x$ successes on 100 attempts given a probability $P(r, m)$ for a single success. At a fixed distance $r$ from the centre we find a $100 \%$ detection rate for bright galaxies, and a drop in the rate at characteristic magnitude $\sim m_{0}$ (Fig. 9). The analytical formula of this function, given by a fit performed with a Fermi function is:

$P(r, m)=\frac{1}{\mathrm{e}^{\frac{m-m_{0}}{c}}+1}$. 


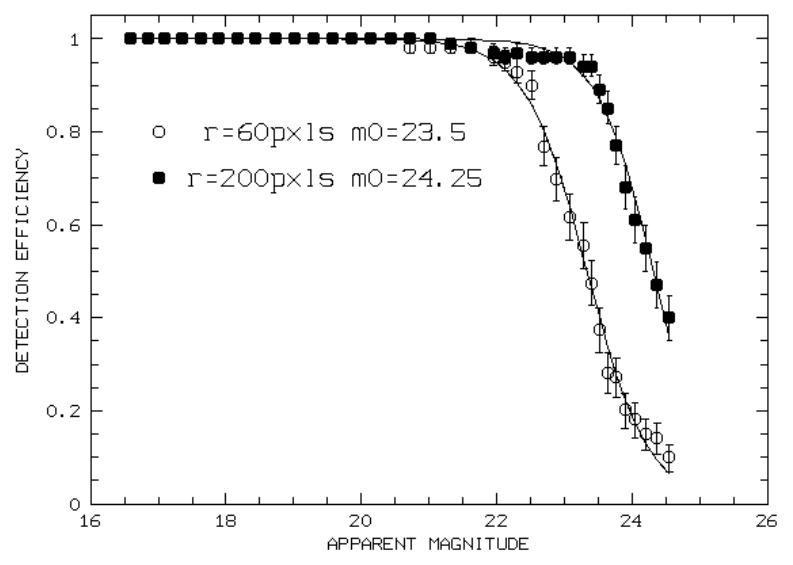

Fig. 9. Bootstrap results at two different distances from the centre of the $\mathrm{cD}$ galaxy are shown. The two different curves are fitted by Fermi-Dirac function with different value of the characteristic magnitude $m_{0}$. Going off centre $m_{0}$ increases: at fixed magnitude, finding a faint galaxy is easier. The uncertainty of the test results is estimate by binomial statistic and $1 \sigma$ level is shown in the figure

We also find that $m_{0}$ depends on the distance $r$. Smaller radii are associated with brighter $m_{0}$. The relationship can be parameterized by an hyperbole

$m_{0}=m_{0}(r)=A-\frac{B}{r}$,

where $A$ and $B$ are slightly different for the 3 filters. As we expect, this relation is affected by background statistical variation and sources crowding. The first steep increase of $m_{0}$ is due to crowding effect of the central part of the cluster and to the $\mathrm{cD}$ halo. The flat shape near an asymptotic value is due to the statistical variations in the background noise. The asymptotic value of $m_{0}$ corresponds to $50 \%$ detection probability independently of any crowding effect and for each filter we assume it as the limiting magnitude value of the catalogue $(24.14,24.46,23.75$, for filter $g, r, i$ respectively).

The test is performed on the raw image, without the exclusion of the bright, extended objects. Indeed, we stress that subtracting the signal from extended sources (see previous section) does not substantially improve the automatic routine detection capability of faint galaxies.

\subsection{Stars and galaxies}

We identify and remove foreground bright stars from the catalogue by using the isophotal magnitude-isophotal radius plane (Fig. 10). In this plane there is a clear distinction between two different populations of sources up to the magnitude $r \simeq 20.75$ : within this range stars have smaller isophotal radius than galaxies at any given magnitude. We cannot classify fainter stars morphologically. Their identification from our data can be achieved only in a statistical way by estimating the contamination level of our sample.
Table 5. By using function $P(r, m)$, we can estimate our sample completeness. Here we give the completeness value of the last three-1magnitude bin for each filter

\begin{tabular}{|l|llc|}
\hline & App. mag. bin & Abs. mag. bin & Compl. (\%) \\
\hline filter $g$ & {$[23.14,24.14]$} & {$[-12.39,-11.39]$} & $67_{-4.8}^{+4.5}$ \\
& {$[22.14,23.14]$} & {$[-13.39,-12.39]$} & $95_{-2.4}^{+1.7}$ \\
& {$[21.14,22.14]$} & {$[-14.39,-13.39]$} & $99_{-0.6}^{+0.9}$ \\
\hline filter $r$ & {$[23.46,24.46]$} & {$[-12.07,-11.07]$} & $68_{-4.7}^{+4.5}$ \\
& {$[22.46,23.46]$} & {$[-13.07,-12.07]$} & $96_{-2.2}^{+1.5}$ \\
& {$[21.46,22.46]$} & {$[-14.07,-13.07]$} & $99_{-0.6}^{+0.9}$ \\
\hline filter $i$ & {$[22.75,23.75]$} & {$[-12.79,-11.79]$} & $71_{-4.6}^{+4.3}$ \\
& {$[21.75,22.75]$} & {$[-13.79,-12.79]$} & $97_{-2.0}^{+1.2}$ \\
& {$[20.75,21.75]$} & {$[-14.79,-13.79]$} & $99_{-0.6}^{+0.9}$ \\
\hline
\end{tabular}

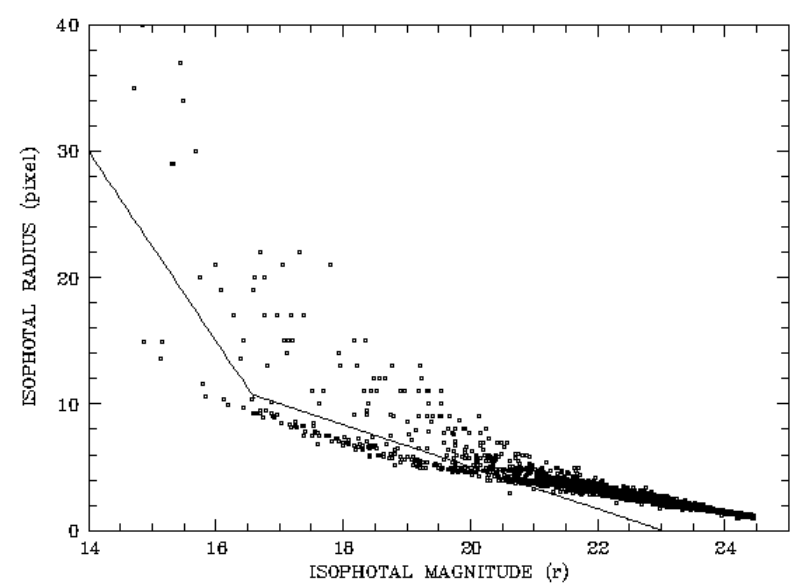

Fig. 10. The isophotal magnitude-isophotal radius plane: we can easily identify 279 bright stars up to $r=20.75$. At fainter magnitudes our data do not allow us to classify morphologically the sources of our sample. The continuous line mark the separation between the star and the galaxy fields

In the bright part of the catalogue $(14.0<\operatorname{mag}<20.75)$ we identify 279 stars. In the remaining part of the catalogue, we expect to have 290 foreground faint stars (Table 6 ), about $15 \%$ of the total of faint sources (Robin et al. 1995). The star contamination level falls under $10 \%$ if we limit our analysis to the "sequence" galaxies sample (see next section).

Table 6. Number of faint, unclassified, stars expected within our catalogue, divided into 1 magnitude bins. First row refers to the whole sample, second row refers to "sequence" colours. The last column reports ratios between stars and galaxies: contamination level of the whole sample is about $15 \%$, whereas sequence galaxies contamination is under $10 \%$

\begin{tabular}{|l|cccc|c|}
\hline MAG & 21.25 & 22.25 & 23.25 & 24.25 & TOTAL \\
\hline stars & 56 & 75 & 94 & 65 & $290 / 1867$ \\
stars & 13 & 7 & 13 & 14 & $47 / 530$ \\
\hline
\end{tabular}




\subsection{Error estimate}

The Poissonian uncertainty is the largest source of error in our photometric measurements and can be estimated by comparing independent magnitude measurements of the same objects. In our sample, we have independent photometric measurements of the objects belonging to the intersections of two adjoining fields. As shown in Table 7, they represent a statistically significant subsample.

Table 7. Number of objects belonging to the intersection of different fields

\begin{tabular}{|l|ccc|}
\hline Filter & $g$ & $r$ & $i$ \\
\hline Field $1 \cap$ Field 0 & 91 & 141 & 128 \\
Field $1 \cap$ Field 2 & 95 & 126 & 107 \\
\hline
\end{tabular}

Starting from the Poissonian statistics, due to the errors propagation law, for the magnitude uncertainty, we expect

$\sigma(m)=$ const $10^{0.2 m}$,

where the constant is given by the characteristics of the electronics. At magnitude 22 we estimate that the uncertainty of the photometric measures $\sigma_{22}$ is $0.20,0.19$, 0.22 magnitude for filter $g, r, i$ respectively. Then, for each magnitude $m$ we can draw $\sigma_{m}$ as

$\sigma(m)=\sigma_{22} 10^{0.2(m-22)}$,

which is the uncertainty of our photometric measures as function of the magnitude.

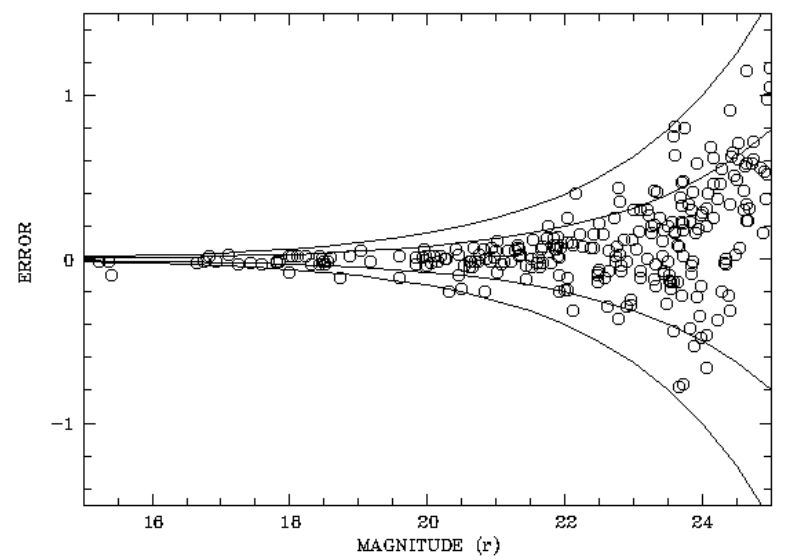

Fig. 11. Differences between $r$ magnitude measurements of the same objects performed from two frames. Plotted against magnitude, they show the expected exponential slope. 1 and $2 \sigma_{m}$ level curves are shown

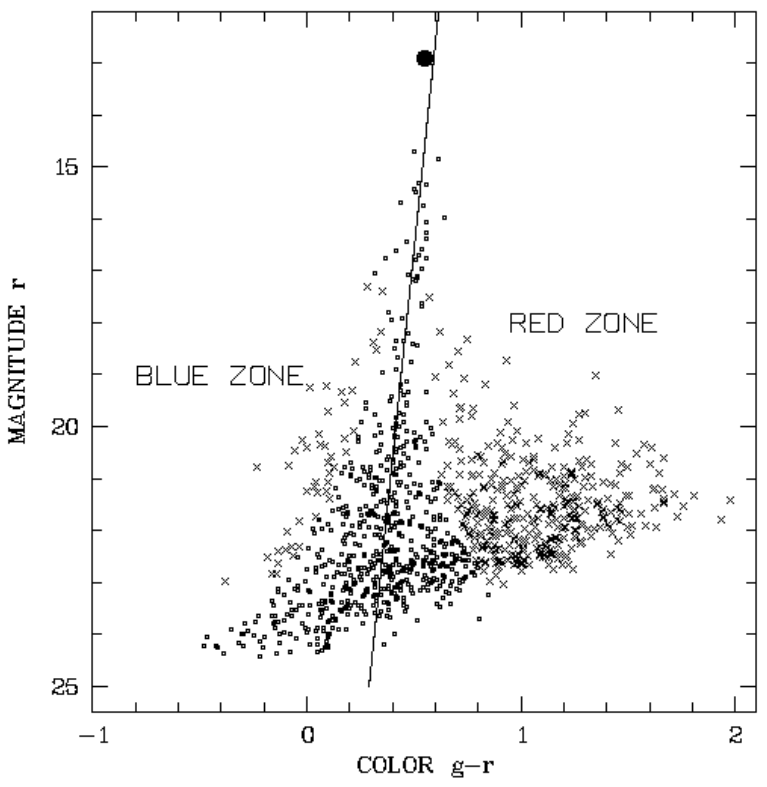

Fig. 12. We split up the plane in three different regions on the basis of the Colour-Magnitude Relation. Using Metcalfe et al. (1994) terminology the three regions are defined as the "blue", "the sequence" and the "red". We identify 637 galaxies within the sequence zone (little squares), 371 in the red zone and 47 in the blue one. The filled circle at $r \sim 13$ refers to $\mathrm{cD}$ galaxy isophotal magnitude and core index colour (see next subsection)

\subsection{Description of the catalogue}

The derived catalogue consists of 2355 objects: 2076 are classified as galaxies, 956 galaxies have magnitude measures in all three filters, 1081, 2055, 1500 galaxies have magnitude values below the filter $g, r, i$ limit, respectively. We estimate $g-r$ colours of 1058 galaxies and $g-i$ of 955 galaxies. The whole catalogue is available in electronic form (http://www.merate.mi.astro.it/ molinari/A496cat.dat), while in Table 8 the list of the 40 brightest galaxies is reported, and in Table 9 we summarise the statistics of the catalogue of galaxies. In the different columns we list:

- (1) ID number of the object;

- (2) EAST and SOUTH coordinates, in arseconds, with respect to the centre of the $\mathrm{cD}$ galaxy;

- (3) $g, r, i$ isophotal magnitudes, each computed down to the threshold quoted in the previous section;

- (4) $g, r, i$ isophotal radius;

- (5) $g-r$ and $g-i$ colours index computed through a 1.5, 3 or 5 pixel aperture photometry, depending on the computed $r$ isophotal radius;

- (6) classification of the object as star or galaxy. 
Table 8. A subsample of the photometric catalogue (http://www.merate.mi.astro.it/ molinari/A496-cat.dat) reporting the 40 brightest objects in the complete list is presented. The luminosity sorting has been made in the $r$ filter. The ID number refers to the position in the whole catalogue

\begin{tabular}{|c|c|c|c|c|c|c|c|c|c|c|c|}
\hline ID & $x$ & $y$ & $g$ & $r$ & $i$ & $R_{g}$ & $R_{r}$ & $R_{i}$ & $g-r$ & $g-i$ & note \\
\hline 1429 & -652.6 & 419.8 & 15.22 & 14.70 & 14.54 & 32.0 & 35.0 & 31.0 & 0.50 & 0.68 & star \\
\hline 661 & -553.3 & 23.8 & 15.41 & 14.84 & 14.71 & 36.0 & 40.0 & 34.0 & 0.61 & 0.76 & star \\
\hline 2251 & -856.6 & 939.4 & 14.81 & 14.87 & 14.87 & 13.5 & 14.9 & 12.2 & -0.17 & -0.10 & \\
\hline 1525 & -292.9 & 501.1 & 15.79 & 15.13 & 14.95 & 11.6 & 13.6 & 12.3 & 0.66 & 0.83 & \\
\hline 1061 & -640.4 & 193.7 & 15.66 & 15.15 & 15.25 & 12.0 & 15.0 & 11.7 & 0.47 & 0.33 & \\
\hline 748 & -272.2 & 55.5 & 15.83 & 15.30 & 15.17 & 26.0 & 29.0 & 25.0 & 0.52 & 0.65 & star \\
\hline 249 & -240.6 & -146.6 & 15.89 & 15.34 & 15.29 & 26.0 & 29.0 & 25.0 & 0.56 & 0.60 & star \\
\hline 1448 & -286.8 & 440.7 & 15.99 & 15.44 & 15.32 & 31.0 & 37.0 & 30.0 & 0.50 & 0.68 & star \\
\hline 968 & -631.8 & 152.6 & 15.98 & 15.48 & 15.42 & 32.0 & 34.0 & 29.0 & 0.50 & 0.58 & star \\
\hline 114 & -587.8 & -198.7 & 16.15 & 15.69 & 15.55 & 27.0 & 30.0 & 27.0 & 0.43 & 0.61 & star \\
\hline 935 & 47.3 & 138.5 & 16.27 & 15.76 & 15.59 & 18.0 & 20.0 & 19.0 & 0.54 & 0.71 & star \\
\hline 1543 & -437.4 & 514.5 & 16.11 & 15.80 & 15.80 & 11.0 & 11.6 & 9.9 & 0.33 & 0.33 & \\
\hline 573 & -149.6 & -22.6 & 16.19 & 15.83 & 15.81 & 10.1 & 10.6 & 10.3 & 0.43 & 0.43 & \\
\hline 1365 & -418.3 & 373.6 & 16.58 & 15.99 & 15.87 & 20.0 & 21.0 & 18.0 & 0.64 & 0.79 & star \\
\hline 113 & 159.7 & -199.1 & 16.61 & 16.08 & 15.89 & 17.0 & 19.0 & 18.0 & 0.55 & 0.74 & star \\
\hline 2257 & -1077.9 & 942.2 & 16.32 & 16.12 & 16.18 & 9.9 & 10.4 & 9.0 & 0.19 & 0.16 & \\
\hline 355 & -61.8 & -107.4 & 16.50 & 16.18 & 16.18 & 9.7 & 10.0 & 9.2 & 0.35 & 0.32 & \\
\hline 387 & -25.6 & -93.0 & 16.81 & 16.27 & 16.10 & 14.0 & 17.0 & 15.0 & 0.55 & 0.73 & star \\
\hline 632 & 56.8 & 8.0 & 16.91 & 16.38 & 16.19 & 12.2 & 13.6 & 12.9 & 0.56 & 0.74 & star \\
\hline 71 & -186.1 & -217.7 & 17.81 & 16.42 & 15.35 & 7.4 & 9.7 & 11.8 & 1.43 & 2.47 & \\
\hline 480 & -25.9 & -60.0 & 16.92 & 16.44 & 16.18 & 12.0 & 15.0 & 14.0 & 0.46 & 0.72 & star \\
\hline 1919 & -922.3 & 681.2 & 17.49 & 16.56 & 16.28 & 8.1 & 10.4 & 9.5 & 0.91 & 1.24 & \\
\hline 1200 & -339.8 & 251.2 & 17.12 & 16.58 & 16.52 & 17.0 & 19.0 & 16.0 & 0.53 & 0.62 & star \\
\hline 1280 & -482.8 & 304.7 & 17.74 & 16.59 & 15.89 & 7.8 & 9.3 & 9.6 & 1.18 & 1.89 & \\
\hline 1522 & -633.1 & 496.8 & 16.96 & 16.61 & 16.58 & 20.0 & 20.0 & 17.0 & 0.41 & 0.50 & star \\
\hline 1006 & -77.7 & 175.2 & 16.81 & 16.62 & 16.65 & 9.0 & 9.3 & 8.8 & 0.25 & 0.19 & \\
\hline 2032 & -1078.6 & 771.6 & 17.44 & 16.63 & 16.44 & 7.8 & 9.3 & 8.5 & 0.79 & 1.01 & \\
\hline 781 & -236.8 & 69.3 & 16.96 & 16.69 & 16.72 & 8.8 & 9.3 & 8.4 & 0.22 & 0.19 & \\
\hline 2308 & -908.9 & 987.2 & 17.83 & 16.70 & 15.97 & 7.1 & 9.5 & 9.6 & 1.10 & 1.87 & \\
\hline 1511 & -420.9 & 489.4 & 17.24 & 16.70 & 16.61 & 20.0 & 22.0 & 19.0 & 0.52 & 0.67 & star \\
\hline 982 & -135.0 & 159.3 & 17.88 & 16.74 & 16.06 & 7.1 & 9.0 & 9.9 & 1.15 & 1.81 & \\
\hline 1082 & -629.4 & 200.5 & 17.12 & 16.76 & 16.60 & 19.0 & 20.0 & 18.0 & 0.37 & 0.51 & star \\
\hline 925 & 131.5 & 132.0 & 17.31 & 16.76 & 16.61 & 15.0 & 17.0 & 16.0 & 0.55 & 0.70 & star \\
\hline 901 & -516.1 & 121.2 & 17.14 & 16.78 & 16.77 & 8.7 & 9.5 & 8.5 & 0.34 & 0.34 & \\
\hline 826 & -67.3 & 90.6 & 17.30 & 16.80 & 16.62 & 12.0 & 13.0 & 13.0 & 0.50 & 0.68 & star \\
\hline 981 & -360.6 & 159.0 & 17.24 & 16.86 & 16.85 & 8.7 & 9.3 & 8.2 & 0.32 & 0.33 & \\
\hline 1604 & -1083.8 & 545.4 & 17.86 & 16.87 & 16.52 & 7.7 & 10.1 & 8.9 & 0.95 & 1.34 & \\
\hline 130 & -68.4 & -189.4 & 18.00 & 16.90 & 16.32 & 6.9 & 9.0 & 9.7 & 1.09 & 1.67 & \\
\hline 2082 & -897.2 & 812.4 & 17.13 & 16.93 & 16.96 & 8.6 & 8.9 & 7.6 & 0.17 & 0.22 & \\
\hline 478 & -580.7 & -60.5 & 17.40 & 16.97 & 16.95 & 8.2 & 9.2 & 7.9 & 0.39 & 0.41 & \\
\hline
\end{tabular}

\section{Abell 496 photometric properties}

In this section we analyse the general properties of the cluster. We examine closely the following points. First, by means of the Colour-Magnitude Relation, we select the main, early type, component of the cluster population. Second, we estimate the projected spatial distribution of the different types of galaxies and we measure the core radius of the cluster as tracked by bright galaxies. Third, we analyze the photometric properties of the $\mathrm{cD}$ central galaxy. Fourth, we study the distribution of galaxy colour as function of their position within the cluster core.

\subsection{The colours of the galaxies}

On the $r /(g-r)$ plane (Fig. 12) we emphasize the narrow sequence of the linear Colour-Magnitude Relation $(C M R)$ : the sequence defines the locus of early type galaxies of the cluster within the plane (Visvanathan \& Sandage 1977; Arimoto \& Yoshii 1987). The continuous line is determined by fitting the locus of points as defined by elliptical galaxies brighter than magnitude 18, excluding the $\mathrm{cD}$ galaxy. The equation derived by the best fit

$C M R(r)=-0.025 r+0.914$

has been extrapolated to the limiting magnitude of the frame. The slope of the $C M R$ is consistent with that 

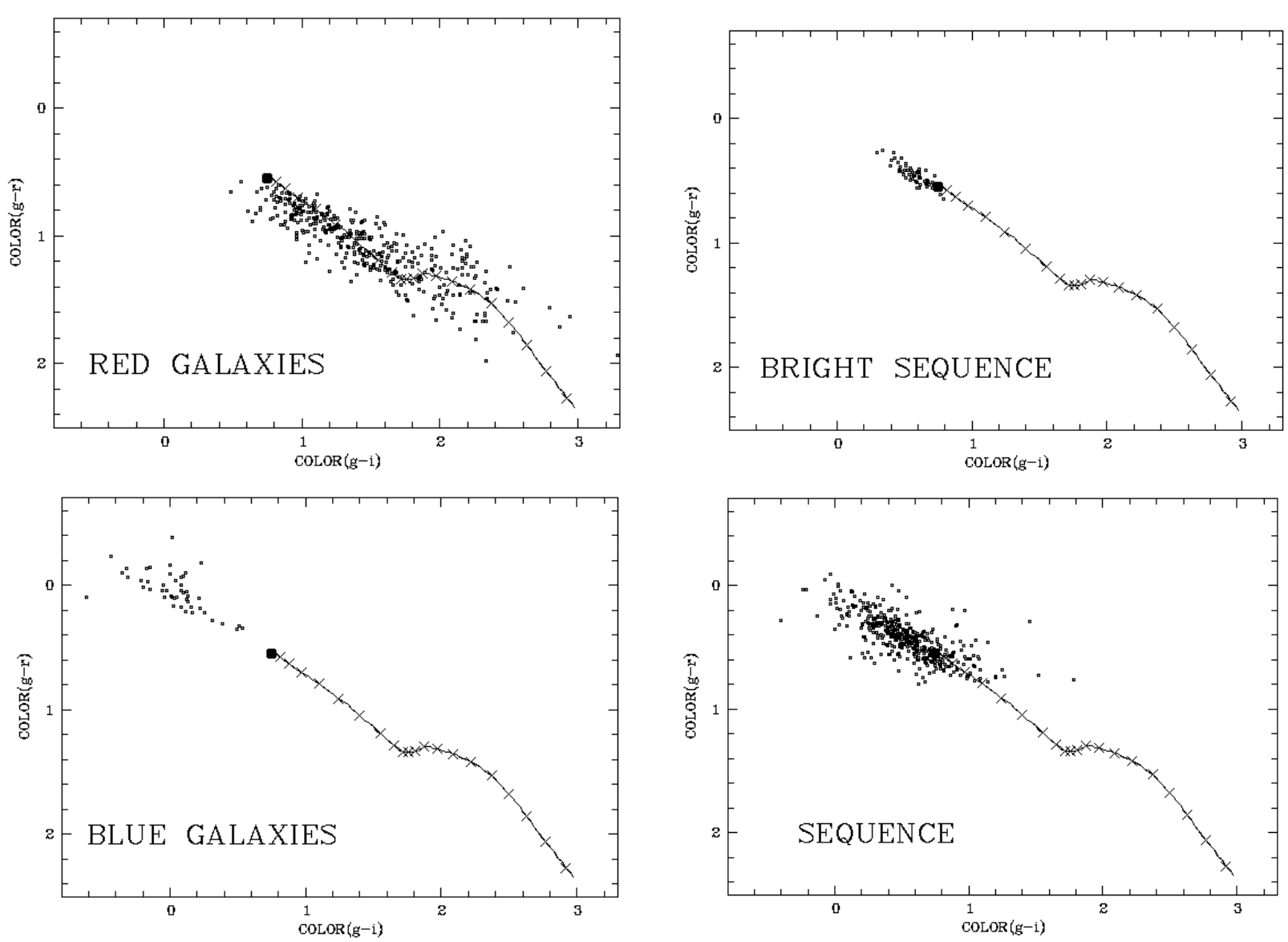

Fig. 13. Colour-colour planes. Our data are superimposed on the expected colours of elliptical galaxies at different red-shifts: each cross on the continue line represents a 0.05 red-shift variation. The filled square represents the cD galaxy, perfectly placed on the theoretical path at red-shift 0.03. Redder galaxies show expected colours of elliptical galaxies at higher red-shift. Sequence galaxies are slightly bluer than $\mathrm{cD}$ galaxy with dispersion increasing with the magnitude (see Fig. 12) Finally, blue galaxies have colours unmatchable with the early type galaxy colours

Table 9. Statistics of the catalogue of galaxies; 279 classified bright stars are included in the catalogue, but not in the present summary table. At faint magnitudes $(>20.75)$ we expect $15 \%$ of the entries are foreground stars. In parentheses absolute magnitude limits are reported assuming $H_{0}=$ $50 \mathrm{~km} / \mathrm{s} / \mathrm{Mpc}$

\begin{tabular}{|l|cc|c|}
\hline Skill & Min. & Max & Gal. enters \\
\hline$\delta_{2000}$ & $-13^{\circ} 32^{\prime} 24^{\prime \prime}$ & $-13^{\circ} 11^{\prime} 26^{\prime \prime}$ & 2077 \\
$\alpha_{2000}$ & $4^{\mathrm{h}} 32^{\prime} 48^{\prime \prime}$ & $4^{\mathrm{h}} 33^{\prime} 49^{\prime \prime}$ & 2077 \\
mag $g$ & $12.64(-22.93)$ & $24.14(-12.28)$ & 1081 \\
mag $r$ & $12.04(-22.62)$ & $24.47(-11.96)$ & 2055 \\
mag $i$ & $11.88(-22.55)$ & $23.75(-12.67)$ & 1500 \\
Col. $g-r$ & -0.50 & 1.98 & 1059 \\
Col. $g-i$ & -0.61 & 3.28 & 956 \\
\hline
\end{tabular}

estimated by Visvanathan \& Sandage (1977) for the Virgo cluster (see their Table 1 and Figs. 1 and 2) and very similar to the estimates given by Garilli et al. (1996). The cD galaxy fits quite nicely the locus of the elliptical galaxies and the $C M R$ relation.
Several authors have used the $C M R$ to define cluster members (Metcalfe et al. 1994; Biviano et al. 1995; Secker 1996; Lopez-Cruz et al. 1997; De Propris \& Pritchet 1998; Molinari \& Smareglia 1998) since, by so doing, the contamination, due to the background galaxies, is largely reduced. Given the analytical formula of the linear relation $C M R(r)$, determined above, we define the "sequence zone" as the colour-magnitude plane region inside the curves

$(g-r)(r)=C M R(r) \pm\left(\sqrt{\sigma_{g}(g)^{2}+\sigma_{r}(r)^{2}}+0.06\right)$,

where we take into account photometric uncertainty at $1 \sigma$ level (see Sect. 4.4) and the inherent dispersion of the relation (estimated upon the most luminous galaxies). The plane redward of the sequence (red zone) is expected to be mainly populated by higher red-shift galaxies, while the blueward zone is likely the locus of cluster and foreground late-type galaxies.

To further clarify this concept of likely membership we plot our data in the colour-colour plane, $g-r$ versus $g-i$ (Fig. 13). The continuous line in the plane represents the 

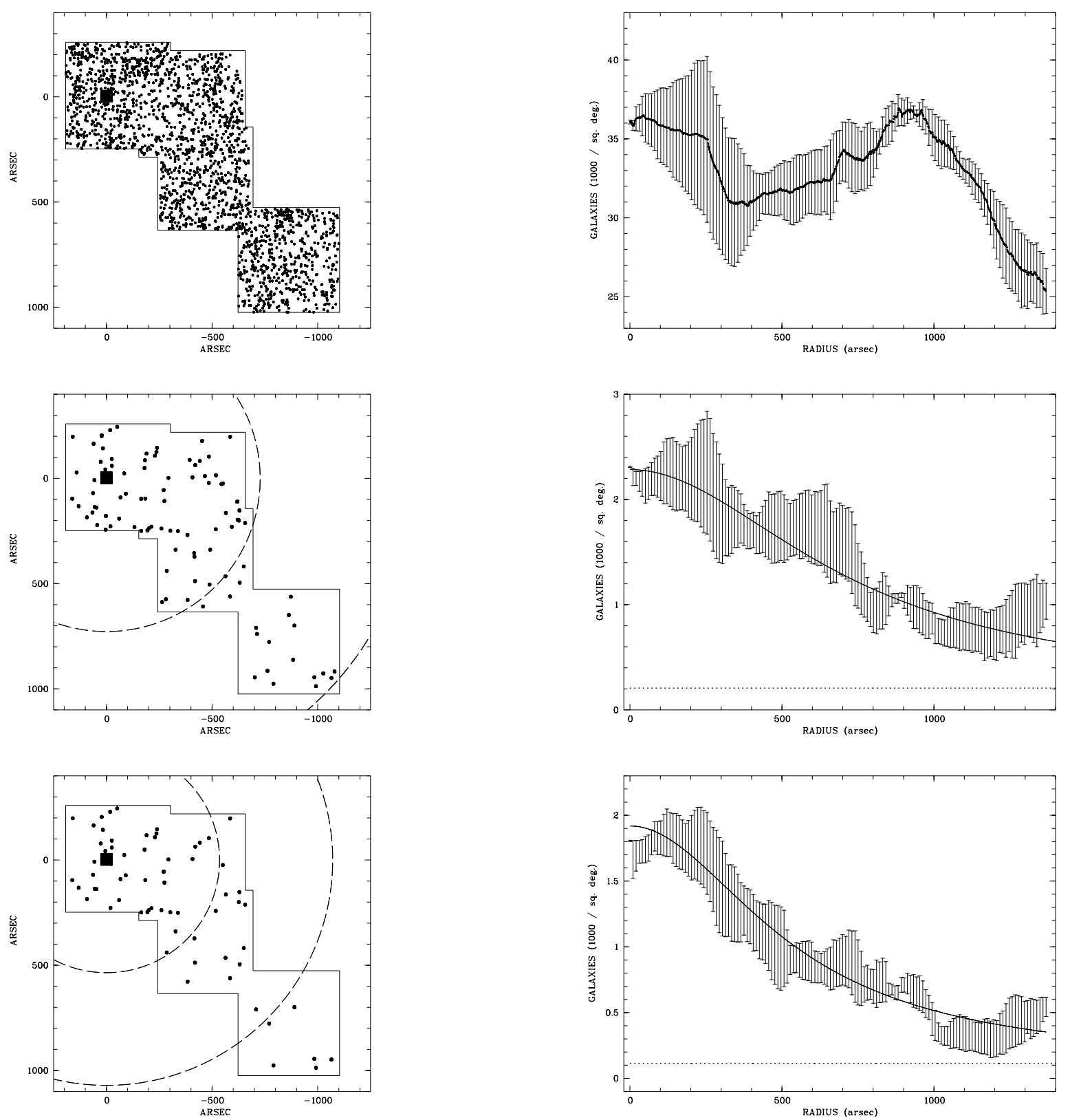

Fig. 14. Projected spatial distribution of all (upper panel), bright $(r \leq 20.0)$ galaxies (central panel), and bright sequence galaxies (lower panel). We fit bright galaxies distributions with King functions and we show the two different core radius best values. Comparison between the upper and central panel suggests a luminosity segregation effect; comparison between central and lower panel suggest a colour segregation effect. The density profile is obtained as the average of 36 profiles, and the errors correspond to 1 standard deviation of the 36 values distribution. In the left panel the dotted lines show 1 e 2 core radius

locus of points defined by elliptical galaxies at different redshifts according to the models of Buzzoni et al. (1993). These plots are consistent with the previous discussion: a) the $\mathrm{cD}$ galaxy, filled square, is near the expected location of an E galaxy at the cluster redshift, b) galaxies located in the red zone of Fig. 13 are displayed along the sequence of higher redshift ellipticals, and c) blue galaxies do not match the redshift sequence for elliptical galaxies.

\subsection{Spatial distribution}

The strategy we adopt for the observations has the advantage of allowing measuring fields at a rather large distance, about 2700 pixels $(\sim 1275 \mathrm{kpc})$ from the cluster centre in a reasonable amount of telescope time. On the other hand we are forced to select an ad hoc radial direction. That is we are more sensitive to cluster and background field density fluctuations. We proceed as follows. First, we build 

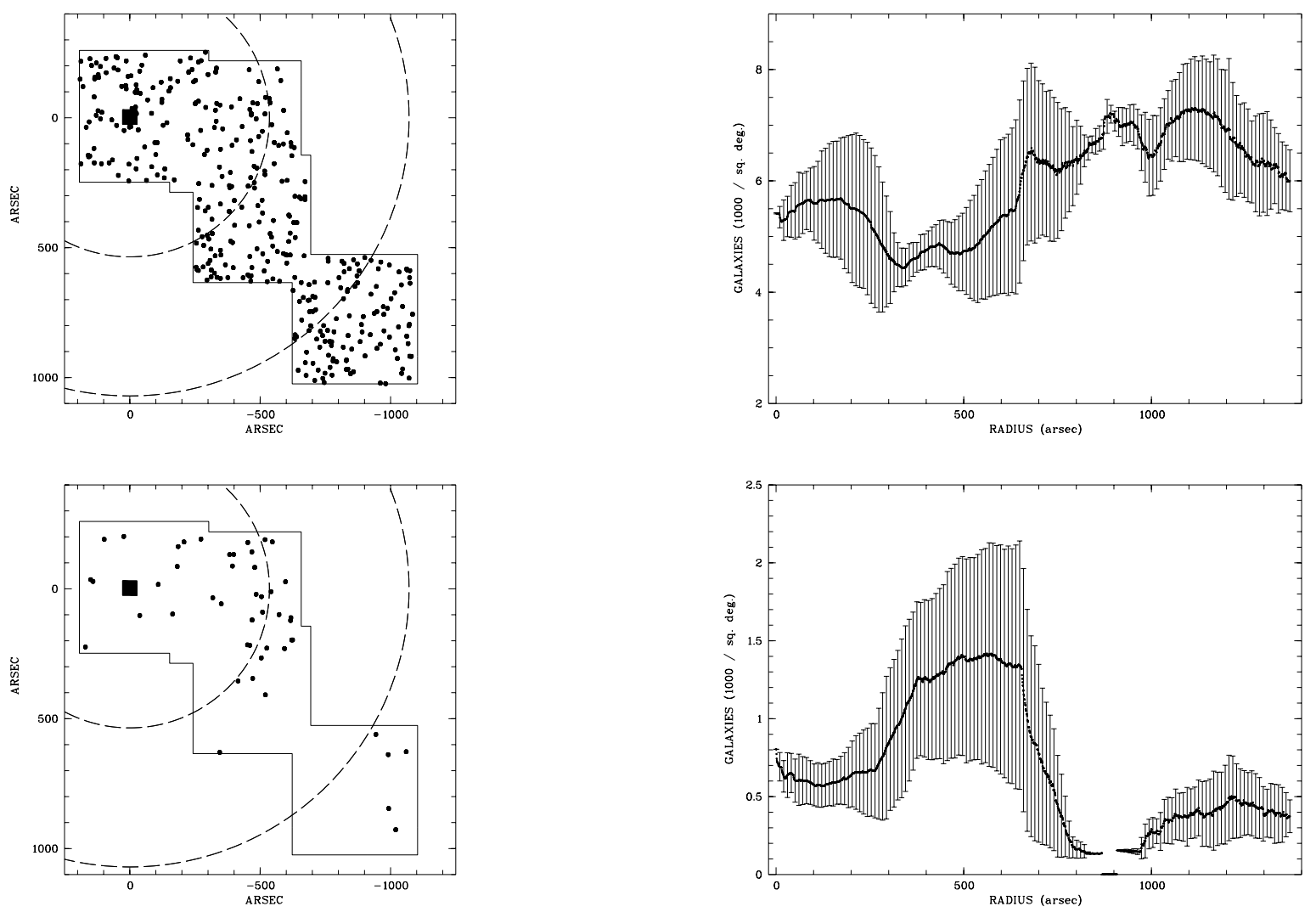

Fig. 15. Spatial distribution of red galaxies (upper panel) and blue galaxies (lower panel) as labelled on the magnitude-colour plane. Red galaxies do not show any particular behaviour linked to cluster structure. Blue galaxies remarkably crowd at 1 core radius distance from the centre of the cluster

the density frame relative to the whole mosaic. Then we divide the density frame in 36 circular sectors centred on the cluster centre and average the contribution of each segment at fixed radius going from the centre to the external limit of the mosaic. The whole sample mean radial surface density profile (Fig. 14, upper panel) does not clearly make evident the excess of galaxies defining the cluster.

Due to the segregation effect of the most luminous galaxies, $r<20.0$, a King profile well fits the density profile at these magnitudes (Fig. 14 central panel, and Table 10). The sequence galaxies as defined by the $C M R$, with $r<20.0$, present a higher central concentration as indicated by the smaller core radius (Table 10). This is also to be expected in a relaxed cluster since the $C M R$ sequence has been defined by using the bright elliptical cluster galaxies.

Galaxies belonging to the red region of colourmagnitude plane are identified as galaxies at higher red-shift (see Figs. 12 and 13). Their distribution is homogeneous over the observed field without any link to cluster structure (Fig. 15 upper panel). Galaxies belonging to the blue zone of the colour-magnitude plane are identified as cluster or foreground late type galaxies.
Table 10. Best fit values of King function for the distribution of bright galaxies $(r<20)$

\begin{tabular}{|l|ccc|}
\hline Sample & $\sigma_{0}\left(10^{3} / \mathrm{sq}^{2}\right)$ & $R_{\mathrm{c}}(\operatorname{arcsec})$ & $\sigma_{\infty}\left(10^{3} / \mathrm{sq}^{2}\right)$ \\
\hline ALL & $2.077 \pm 0.2$ & $727_{-38}^{+33}$ & $0.22 \pm 0.02$ \\
SEQUENCE & $1.807 \pm 0.2$ & $497_{-25}^{+22}$ & $0.12 \pm 0.01$ \\
\hline
\end{tabular}

Their projected distribution seems to be influenced by cluster potential: their density abruptly peaks at 1 core radius distance from the cluster centre. This effect has been noticed also in some of the other clusters that we are analysing.

\subsection{The central cD galaxy}

The cD central galaxy is the brightest member of the cluster: it is 2 magnitudes brighter than the second member. In Molinari et al. (1998) its luminosity is regarded as too bright to be consistent with other ellipticals and it is not included in the computation of LF. However, as seen in the previous subsection, the $\mathrm{cD}$ magnitude and colour are consistent with the $C M R$ extrapolated from the population of the bright elliptical galaxies. 
cD galaxies are generally characterised by a surface brightness (SB) profile that falls off more slowly with radius than most elliptical galaxies. In Fig. 16 the profile of the Abell $496 \mathrm{cD}$ galaxy along the major axis is shown up to a distance of $100 \operatorname{arcsec}(\sim 92 \mathrm{kpc})$ from the centre. In this profile the presence of the halo is particularly noticeable, it departs strongly from a de Vaucouleur law (the straight line in the figure). The comparison of the SB profile along the northern major semi-axis $(\mathrm{N})$ with the one along the southern semi-axis (S) (Fig. 16) shows an evident asymmetry. The $\mathrm{N}$ region of the halo exhibits an excess of intensity with respect to the $\mathrm{S}$ in each of the 3 filters in the interval $25-50$ arcsec of distance from the centre. This effect is clearly depicted by the isophotes in Fig. 17. In spite of the large extension of the halo, this is

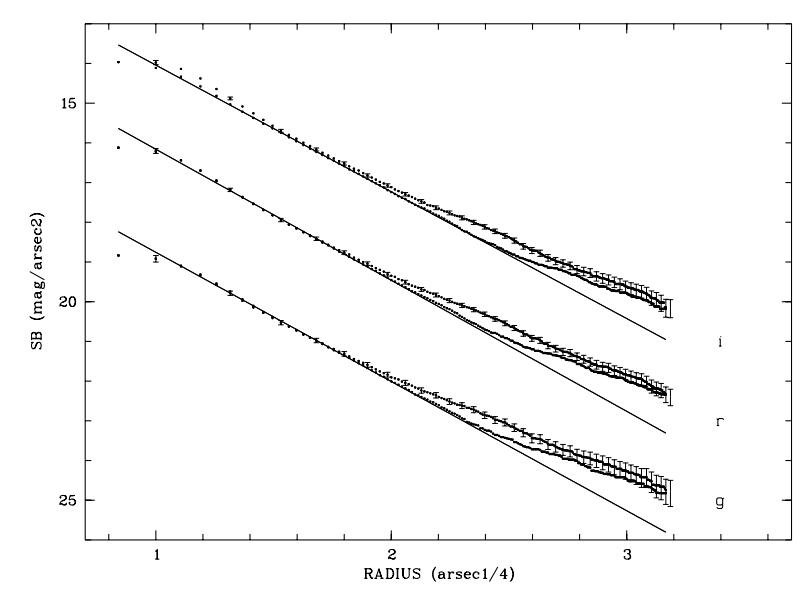

Fig. 16. The intensity profiles of the cD galaxy of Abell 496 along the $\mathrm{N}$ and $\mathrm{S}$ major semi-axis are superimposed (the $r$ and $i$ profiles are shifted of 2 and 4 magnitude to make the figure clearer). The excess of intensity of the northern semi-axis is noticeable in the interval $(25,50)$ arcsec from the centre. The straight lines represents the de Vaucouleur profile

somewhat fainter than the core. After fitting the core by a de Vaucouleur law, we could subtract it from the cD image and estimate the magnitude of the halo. The derived total magnitudes in the three filters are listed in Table 11. As already stated, the luminosity of the core is dominant. The average colour index of the total profile presents a gradient toward the blue moving from the core to the outermost part of the galaxy. This is due to the colour of the halo that is bluer than that of the core. Within the halo itself a difference exists between the colour of the northern hemisphere of higher surface brightness, and the colour of the southern hemisphere. The northern zone is bluer (marked as colour excess in Fig. 18). In other $\mathrm{cD}$ galaxies (see for instance Molinari et al. 1994) the halo has been found redder than the core. Therefore, the characteristics of the halo population are undoubtedly related to the specific history of the $\mathrm{cD}$ under consideration.

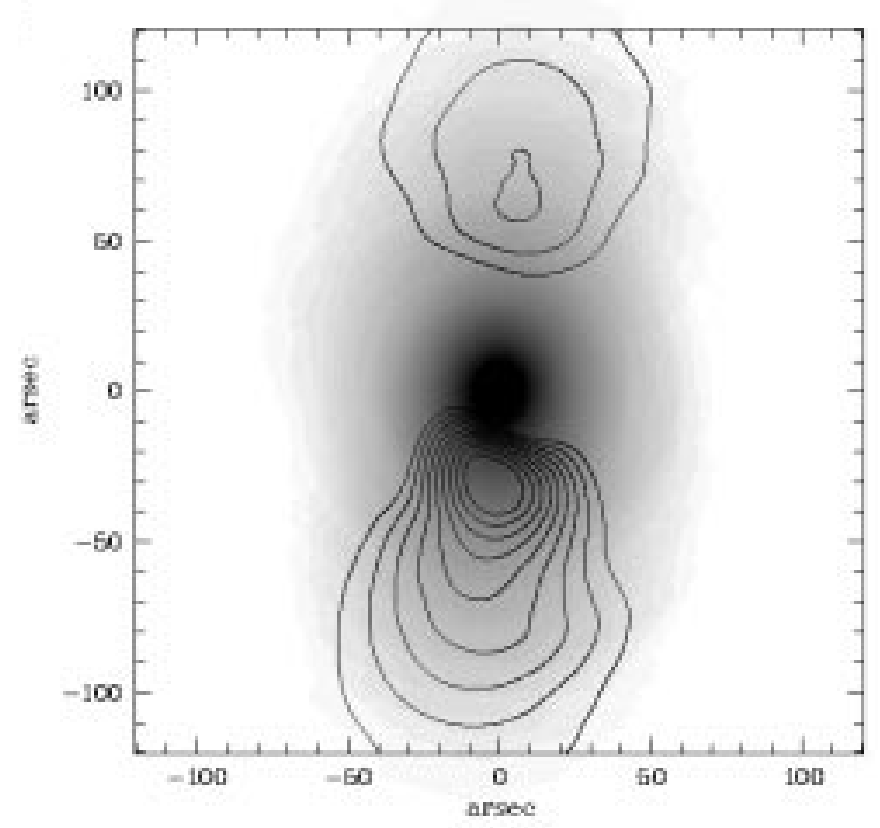

Fig. 17. The filter $r$ halo isophotes are superimposed to the image of the cD galaxy (the North is toward the bottom of the image), the last isophote corresponding to the SB threshold. The asymmetry of the halo emission is clearly evident

\subsection{Colour gradient of the galaxy population}

Finally, the distribution of the $g-r$ colours of the sequence galaxies is analysed as a function of their projected distance from the centre of the cluster. We find a significant correlation relative to the population of faint galaxies.

As partly expected, brighter galaxies tend to dominate in the central region of the cluster. Such galaxies (see also the discussion on the $C M R$ relation) tend to be somewhat redder. Therefore we expect a mild correlation between the cluster integrated colour - defined as the mean colour derived from the galaxy population located at a given distance from the centre - and the distance from the centre. The total gradient expected to be $<0.2$ in $g-r$. On the other hand if we limit ourselves to consider only the dwarf galaxies (bottom of Fig. 19), we do not measure any correlation between the mean galaxy magnitude and the distance from the cluster centre. In spite of this lack of correlation the faint cluster population shows a welldefined colour gradient moving outward from the centre (upper panel of Fig. 19). This effect is significant at a 4 sigma level and unrelated to the $C M R$ relation. Indeed over the small range of magnitude we took into consideration $(18<r<21)$ such an effect would be at most of about $0.1 \mathrm{mag}$, while we observe a gradient of about 0.3 magnitudes.

A very similar result is found by Secker (1996) in Coma cluster; conversely, Hilker et al. (1998) do not find any correlation between the projected distance from the 
Table 11. Photometric parameters of the $\mathrm{cD}$ galaxy

\begin{tabular}{|l|ccccc|}
\hline$F$ & $r_{\mathrm{e}}(\mathrm{arsc})$ & $\mu_{\mathrm{e}}\left(\mathrm{mag} / \mathrm{arsc}^{2}\right)$ & $m_{\text {tot }}$ & $m_{\text {core }}$ & $m_{\text {halo }}$ \\
\hline$g$ & $58.3 \pm 7.5$ & $25.81 \pm 0.12$ & $12.64 \pm 0.03$ & $12.85 \pm 0.04$ & $14.56 \pm 0.07$ \\
$r$ & $51.7 \pm 5.1$ & $25.12 \pm 0.10$ & $12.04 \pm 0.02$ & $12.22 \pm 0.03$ & $13.99 \pm 0.06$ \\
$i$ & $52.7 \pm 4.9$ & $24.96 \pm 0.10$ & $11.88 \pm 0.02$ & $12.05 \pm 0.03$ & $13.97 \pm 0.06$ \\
\hline
\end{tabular}

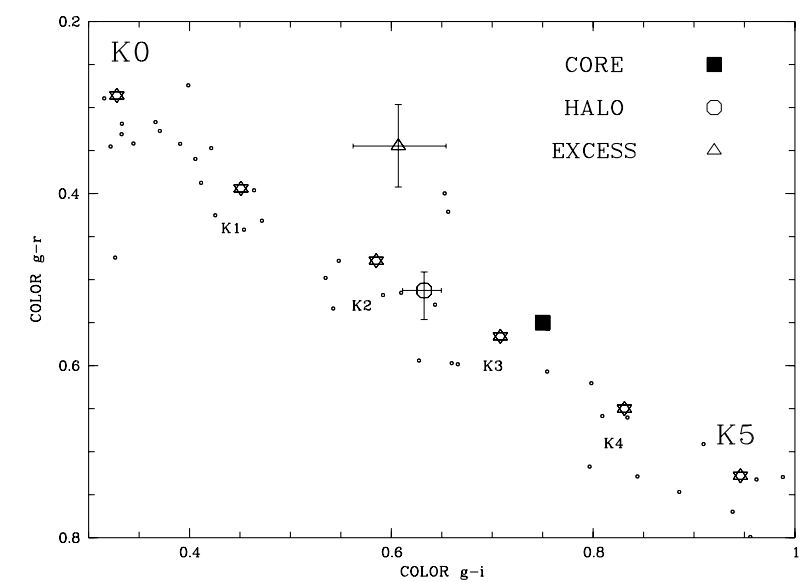

Fig. 18. The average colour index of the three components of the galaxy is shown. They are compared with the expected colours of the stars convoluted from the spectral catalogue of Vilnius et al. (1972) (stars are labelled with the name of spectral class) and also with the colours of the stars of our catalogue (small points)

centre and the colours of dwarf galaxies in the central region of Fornax cluster.

\section{Discussion and conclusion}

We started a project whose main goal is the determination of the cluster Luminosity Function and its relation to the cluster morphology and population. In this paper we describe the procedure used for the analysis of the data using A496 as a test case. The LF of this cluster has been published in Molinari et al. (1998).

In the present study, examination of the space distribution of the blue galaxies reveals a density peak at about a core radius $\sim 500 \operatorname{arcsec}(\sim 0.22 \mathrm{Mpc})$ from the cluster centre. This finding should be related to observation of a blueing of the galaxy population, beginning from the cluster centre to its outskirts (Fig. 19). This phenomenon, demonstrated to be independent from the CMR relation and luminosity segregation, calls for physical differentiation in the galaxy stellar content.

Furthermore, we measure a rather blue cD halo with a remarkable North-South colour asymmetry. This is different from what has been found, e.g., by Molinari et al. (1994) who ascribed the very red cD halo was to a M0like stellar population, implying that any model deserves further consideration.
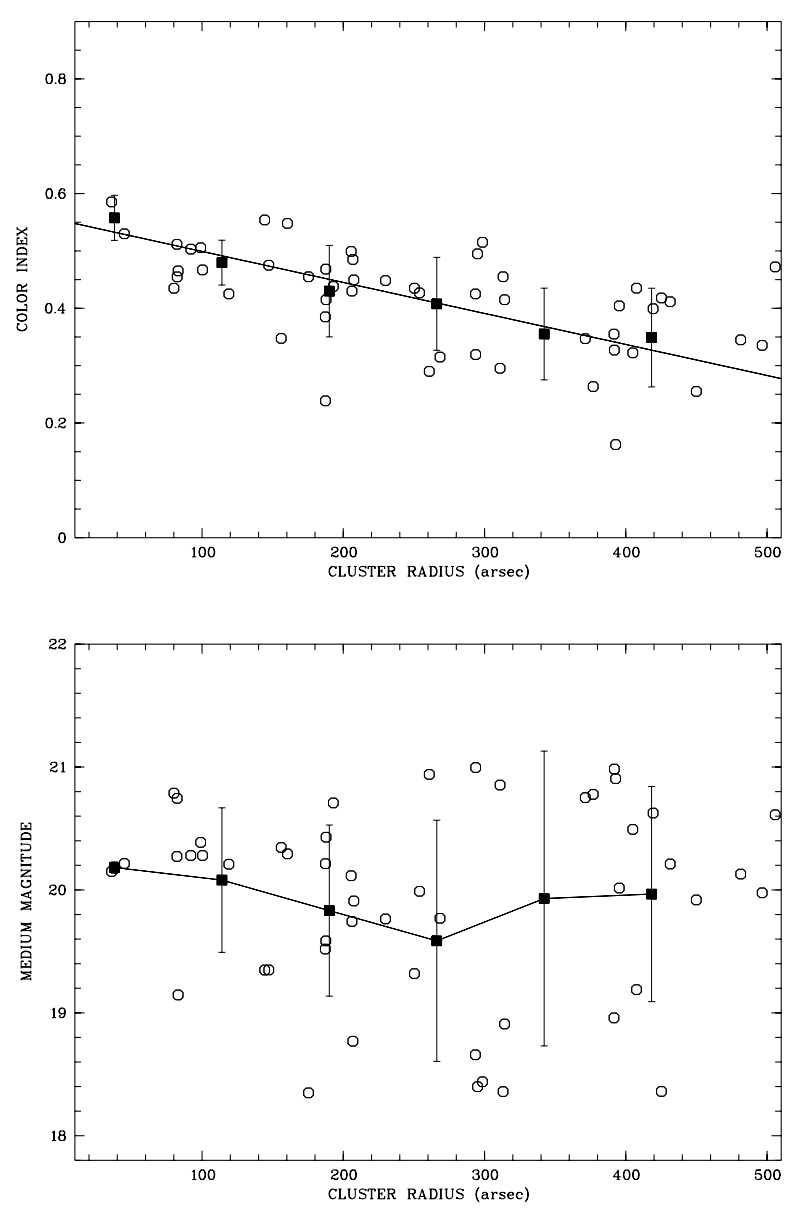

Fig. 19. The average colour index of dwarf sequence galaxies shows a gradient from red to blue going off the centre of the cluster (upper panel). This feature cannot be ascribed to the luminosity+colour segregation: the non correlation between radius cluster and medium magnitude of the dwarf sequence galaxies is shown (lower panel)

Further work is planned to look for these interesting features in other clusters and a detailed discussion on the above results will be given in a forthcoming paper of this series.

Acknowledgements. Thanks are due to K. Sheldt and E. Moretti for some help with English and to CARIPLO Foundation for partial funding. 


\section{References}

Abell G.O., Corwin H.G., Olowin R.P., 1989, ApJS 70, 1

Arimoto N., Yoshii Y., 1987, A\&A 297, 610

Bernstein G.M., Nichol R.C., Tyson J.A., Ulmer M.P., Wittman D., 1995, AJ 110, 1507

Binggeli B., Sandage A., Tamman G.A., 1988, ARA\&A 26, 509

Biviano A., Durret F., Gerbal D., Le Fèvre O., Lobo C., Mazure A., Slezak E., 1995, A\&A 297, 610

Burnstein D., Heiles C., 1982, AJ 87, 1165

Buzzoni A., Chincarini G., Molinari E., 1993, ApJ 410, 499

Buzzoni A., 1995, ApJS 98, 69

Colless M., 1989, MNRAS 237, 799

De Grandi S., Molendi S., Bohringer H., Chincarini G., Voges W., 1997, ApJ 486, 738

De Grandi S., et al., 1999, ApJ 514 (in press)

De Propris R., Pritchet C.J., 1988, AJ 116, 1118

Gunn J.E., Hoessel J.G., Oke J.B., 1986, ApJ 306, 30

Garilli B., Bottini D., Maccagni D., Carrasco L., Recillas E., 1996, ApJS 105, 91

Hilker M., et al., 1999, A\&AS 134, 75

Lopez-Cruz O., Yee H.K.C., Brown J.P., Jones C., Forman W., 1997, ApJL 101, 97L
Metcalfe N., Godwin J.G., Peach J.V., 1994, MNRAS 267, 431

Molinari E., Buzzoni A., Chincarini G., Pedrana M.D., 1995, A\&A 292, 54

Molinari E., Smareglia, 1998, A\&AS 330, 447

Molinari E., Buzzoni A., Chincarini G., 1996, A\&AS 119, 391

Molinari E., Chincarini G., Moretti A., De Grandi S., 1998, A\&A 338, 874 (Paper I)

Robin A., et al., 1995, http://WWW.obsbesancon.fr/ /www/modele/modele_ang.html

Sarazin C.L., 1988, X-ray emission from cluster of galaxies, Astrophysics Series. Cambridge University Press, New York

Secker J., 1996, ApJL 469, L81

Strajzhis V., Sviderskene Z., 1972, Bull. Vilnius astr. Obs. 35, 1

Thuan T.X., Gunn J.E., 1976, PASP 88, 543

Trentham N., 1997, MNRAS 290, 334

Trentham N., 1998, MNRAS 294, 193

Visvanathan N., Sandage A., 1977, ApJ 216, 214

Wade R.A., Hoessel J.G., Elias J.H., Huchra J.P., 1979, PASP 91, 35

Withmore B.C., Gilmore D.M., 1991, ApJ 367, 64

West R.M., Kruszewski A., 1981, Irish Astron. J. 15, 25 